\title{
Resolving Curvature Singularities in Holomorphic Gravity
}

\author{
Christiaan L.M. Mantz • Tomislav Prokopec
}

Received: 8 December 2008 / Accepted: 9 May 2011 / Published online: 4 June 2011

(C) The Author(s) 2011. This article is published with open access at Springerlink.com

\begin{abstract}
We formulate a holomorphic theory of gravity and study how the holomorphy symmetry alters the two most important singular solutions of general relativity: black holes and cosmology. We show that typical observers (freely) falling into a holomorphic black hole do not encounter a curvature singularity. Likewise, typical observers do not experience Big Bang singularity. Unlike Hermitian gravity (Mantz and Prokopec in arXiv:0804.0213v1, 2008), holomorphic gravity does not respect the reciprocity symmetry and thus it is mainly a toy model for a gravity theory formulated on complex space-times. Yet it is a model that deserves a closer investigation since in many aspects it resembles Hermitian gravity and yet calculations are simpler. Our study of light bending and gravitational waves in weak holomorphic gravitational fields strongly suggests that holomorphic gravity reduces to general relativity at large distance scales.
\end{abstract}

Keywords Alternative gravity theories

\section{Introduction}

The existence of singularities in Einstein's well tested theory of general relativity have puzzled many physicists since their discovery. It is a goal of (and motivation for) theories as quantum gravity to remove them. We propose generalizations of general

C.L.M. Mantz · T. Prokopec $(\bowtie)$

Department of Physics and Astronomy, Vrije University, 1081 HV Amsterdam, The Netherlands e-mail: T.Prokopec@uu.nl

\section{C.L.M. Mantz}

e-mail: CMantz@few.vu.nl

C.L.M. Mantz · T. Prokopec

Institute for Theoretical Physics, University of Utrecht, Princetonplein 5, P.O. Box 80.006, 3508 TA

Utrecht, The Netherlands 
relativity which to a great extent ease the singularity problems already at the classical level of the theory. Singularities of general relativity are typically manifest as divergences of some curvature invariant and are ubiquitous in general relativity $[2,3]$.

Consider, for example, the Big Bang singularity. A universe undergoing a power-law expansion expands with a scale factor, $a \sim t^{1 / \epsilon}$, where $\epsilon=-\dot{H} / H^{2}=$ constant $\geq 0$ denotes the 'slow roll' parameter, $H=\dot{a} / a$ is the Hubble expansion rate and $d o t$ denotes a derivative with respect to physical time $t$. The Ricci scalar $R$ then diverges as $t \rightarrow 0$ (corresponding to the time when matter density diverges),

$$
R=6(2-\epsilon) H^{2}=\frac{6(2-\epsilon)}{\epsilon^{2} t^{2}} \rightarrow \infty \quad \text { as } t \rightarrow 0 .
$$

Analogously, the Schwarzschild metric is singular when the coordinate radius $r=$ $\|\vec{x}\| \rightarrow 0$ (corresponding to the place where all of the mass is concentrated), resulting in the curvature singularity of the Riemann tensor,

$$
R_{\mu \nu \rho \sigma} R^{\mu \nu \rho \sigma}=\frac{48 G_{N}^{2} M^{2}}{c^{4} r^{6}} \rightarrow \infty, \quad \text { as } r \rightarrow 0,
$$

where $M$ denotes the black hole mass, $G_{N}$ the Newton constant and $c$ the speed of light.

On complex manifolds space-time coordinates $x^{\mu}$ get complexified as [1], ${ }^{1}$

$$
x^{\mu} \rightarrow z^{\mu}=x^{\mu}+i y^{\mu}, \quad y^{\mu} \equiv \frac{G_{N}}{c^{3}} p^{\mu},
$$

where $p^{\mu}$ denotes the momentum-energy part of an observer's frame (e.g. of a test particle) with respect to the underlying holomorphic space. For an observer whose momentum coordinate $p^{\mu}$ changes slowly along a geodesic, $p^{\mu}$ reduces to the particle's momentum-energy, $p^{\mu} \rightarrow m u^{\mu}$, where $u^{\mu}=d x^{\mu} / d \tau$ and $\tau$ denotes a proper time. In this work the coordinate $p^{\mu}$ is generally not equal to the familiar momentumenergy vector of general relativity. ${ }^{2}$ Based on the holomorphy symmetry, we expect that the singularities (1-2) appear as some power of

$$
\frac{1}{z}
$$

(and possibly its complex conjugate), where $z \rightarrow z^{0}=x^{0}+i y^{0}=x^{0}+i\left(G_{N} / c^{4}\right) E$ for the Big Bang singularity (1) and $z \rightarrow\|\vec{z}\|=\|\vec{x}\|+i\|\vec{y}\|=\|\vec{x}\|+i\left(G_{N} / c^{3}\right)\|\vec{p}\|$ for the black hole singularity (2). Near singularities the observed space-time curvature is typically proportional to a power of the real part of (4),

$$
\mathfrak{R}\left[\frac{1}{z}\right]=\frac{x}{x^{2}+y^{2}},
$$

\footnotetext{
${ }^{1}$ For the sake of simplicity here we drop a factor $1 / \sqrt{2}$ which we used in Ref. [1] when relating $z^{\mu}$ to $x^{\mu}$ and $p^{\mu}$.

${ }^{2}$ A more elaborate discussion of the physical meaning of the imaginary coordinate $p^{\mu}$ and its low-energy reduction to the momentum-energy four-vector of general relativity, is given in Sects. 10 and 4.
} 
which blows up only when both $x$ and $y$ are simultaneously zero. The aim is now to formulate a generalization of general relativity that yields such complexified singularities and show that for a freely falling observer $x$ and $y$ are almost never zero simultaneously. ${ }^{3}$ In Hermitian gravity [1], the Big Bang singularity can be considered as 'resolved' in the sense that the set of observers moving backwards in time that encounter the Big Bang singularity is of measure zero. So typical observers do not see the singularity. Note that this is not the case in general relativity, where all backward-moving observers eventually hit the Big Bang singularity. In this paper we present a new theory, holomorphic gravity, in which also typical observers falling into a Schwarzschild black hole do not encounter a singularity. We believe that this resolution of singularities is a generic feature of gravity theories formulated on complex spaces as presented in this work and in Ref. [1], representing one of the principal advantages of complex theories of gravity when compared with Einstein's general relativity. The idea of complexifying general relativity is an old one [4-10]. Yet the possibility that the undelying space is eight-dimensional is quite recent $[1,11-13]$. To our knowledge holomorphic gravity as formulated in this work has up to now not been considered.

\section{Almost Complex Structure}

A natural generalization of general relativity, in order to obtain complex solutions as (4), would be to consider a theory with complex metrics, living on complex manifolds. A general complex metric on a complex manifold is given by

$$
\begin{aligned}
C= & C_{\mu \nu} d z^{\mu} \otimes d z^{\nu}+C_{\mu \bar{\nu}} d z^{\mu} \otimes d z^{\bar{\nu}} \\
& +C_{\bar{\mu} \nu} d z^{\bar{\mu}} \otimes d z^{\nu}+C_{\bar{\mu} \bar{\nu}} d z^{\bar{\mu}} \otimes d z^{\bar{\nu}},
\end{aligned}
$$

where barred indices $z^{\bar{\mu}} \equiv \bar{z}^{\mu}$ denote complex conjugation. Hermitian gravity [1] is formulated on a Hermitian manifold endowed with a Hermitian metric, defined as follows

$$
C_{p}\left(J_{p} Z, J_{p} W\right)=C_{p}(Z, W),
$$

where the action of the almost complex structure operator, $J$, on the basis vectors of the complexified tangent space is given by

$$
J_{p}\left(\frac{\partial}{\partial z^{\mu}}\right)=i \frac{\partial}{\partial z^{\mu}}, \quad J_{p}\left(\frac{\partial}{\partial \bar{z}^{\mu}}\right)=-i \frac{\partial}{\partial \bar{z}^{\mu}} .
$$

A Hermitian metric is a complex metric which has-as a consequence of the symmetry requirement (6) — vanishing $C_{\mu \nu}$ and $C_{\bar{\mu} \bar{\nu}}$ components:

$$
C=C_{\mu \bar{\nu}} d z^{\mu} \otimes d z^{\bar{\nu}}+C_{\bar{\mu} \nu} d z^{\bar{\mu}} \otimes d z^{\nu}
$$

\footnotetext{
${ }^{3}$ The meaning of 'almost never' is made more precise below.
} 
The requirement that the complex metric satisfies Bianchi identities lead us in [1] to the conclusion that (6) can be consistently imposed on the metric only at the level of the equations of motion (on-shell), while at the level of the action (off-shell) all complex metrics of the form (5) are in fact allowed. Thus studying holomorphic gravity allows one also to rectify the differences between the full complex theory (which, apart from holomorphy on vielbeins, has no additional symmetry requirements) and hermitian gravity.

One of the reasons why one wants to study a theory of gravity that is invariant under the operation of $J$, is that the commutation relations of quantum mechanics are invariant under its action, which seems an invitation for quantization (recall that the $y^{\mu}$ coordinate in (3) is identified with the momentum-energy coordinate, $p^{\mu}$, in the commutation relations). Constructing a geometric theory of gravity on complex manifolds which admits an almost complex structure operator is reminiscent by an old idea of Max Born, according to which quantum theory of gravity should respect a reciprocity symmetry $[14,15]$; for a more modern implementation of Born's idea see $[16,17]$.

We can also consider the theory which is anti-symmetric under the action of the almost complex structure operator, in the sense that the holomorphic metric is defined in the following manner

$$
C_{p}\left(J_{p} Z, J_{p} W\right)=-C_{p}(Z, W) .
$$

The holomorphic metric can then be written as

$$
C=C_{\mu \nu} d z^{\mu} \otimes d z^{\nu}+C_{\bar{\mu} \bar{\nu}} d z^{\bar{\mu}} \otimes d z^{\bar{\nu}}
$$

where the component $C_{\mu \nu}\left(C_{\bar{\mu} \bar{\nu}}\right)$ is (anti-)holomorphic, which simplifies calculations. In the remainder of this paper we construct a theory of gravity, based on the holomorphic metric (9). In the flat space limit the holomorphic metric is invariant under the complexified Lorentz group, $S O(1,3 ; \mathbb{C})$, while the line element is invariant under the complexified inhomogeneous Lorentz group (the complexified Poincaré group) $I S O(1,3 ; \mathbb{C})$.

\section{The Holomorphic Metric}

The holomorphic line element ${ }^{4}$ is defined in the following manner

$$
d s^{2}=d z^{\mu} C_{\mu \nu} d z^{\nu}+d z^{\bar{\mu}} C_{\bar{\mu} \bar{\nu}} d z^{\bar{\nu}}
$$

\footnotetext{
${ }^{4}$ One could instead consider the line element $d s_{c}^{2}=C_{\mu \nu} d z^{\mu} d z^{v}$, which is by definition left invariant by the $I S O(1,3, \mathbb{C})$ group. The reason why we present $(10)$ as the line element is that this line element corresponds to the observed proper length and hence extends general relativity in a more natural way than $d s_{c}^{2}=C_{\mu \nu} d z^{\mu} d z^{\nu}$. We find below that we need to analytically extend the proper time variable to uniquely specify solutions to the geodesic equation. This analytic extension corresponds a complex proper time $\tau$, which is exacted by $d s_{c}^{2}=C_{\mu \nu} d z^{\mu} d z^{v} \equiv-c^{2} d \tau^{2}$.
} 
which can be written in eight dimensional notation

$$
\begin{aligned}
d s^{2} & =d z^{T} \cdot \boldsymbol{C} \cdot d z=\left(d z^{m}\right)^{T} \boldsymbol{C}_{m n} d z^{n} \\
& =\left(d z^{\mu}, d z^{\bar{\mu}}\right)\left(\begin{array}{cc}
C_{\mu \nu} & 0 \\
0 & C_{\bar{\mu} \bar{\nu}}
\end{array}\right)\left(\begin{array}{l}
d z^{\nu} \\
d z^{\bar{\nu}}
\end{array}\right),
\end{aligned}
$$

where the Latin indices can take the values $0,1, \ldots, d-1, \overline{0}, \overline{1}, \ldots, \overline{d-1}$, the Greek indices run in the range $0,1, \ldots, d-1$ and $d$ denotes the complex dimension of the complex manifold. The entries of the metric $\boldsymbol{C}_{m n}$ are functions of holomorphic and antiholomorphic vielbeins defined as follows ${ }^{5}$

$$
\begin{aligned}
C_{\mu \nu} & =e(z)_{\mu}{ }^{a} \eta_{a b} e(z)_{\nu}{ }^{b} \\
C_{\bar{\mu} \bar{\nu}} & =e(\bar{z})_{\mu}{ }^{a} \eta_{a b} e(\bar{z})_{\bar{\nu}}{ }^{b} .
\end{aligned}
$$

The metric components are symmetric under transposition, since they are just an inner product of the vielbein times its transpose. Hence also in eight dimensional notation the metric is symmetric under transposition $\boldsymbol{C}^{T}=\boldsymbol{C}$. We define the $z^{\mu}$ and $z^{\bar{\mu}}$ coordinates in terms of $x^{\mu}$ and $y^{\check{\mu}}$, such that we obtain ${ }^{6}$

$$
z^{\mu}=x^{\mu}+i y^{\check{\mu}}, \quad \frac{\partial}{\partial z^{\mu}}=\frac{1}{2}\left(\frac{\partial}{\partial x^{\mu}}-i \frac{\partial}{\partial y^{\check{\mu}}}\right)
$$

and their complex conjugates. This implies the following decomposition of complex vielbeins in their real, $e_{R}^{a}$, and imaginary, $e_{I}^{a}$, parts in the following manner

$$
\begin{array}{ll}
e_{a}^{\mu}=e_{R_{a}^{\mu}}^{\mu}+i e_{I_{a}}^{\check{\mu}}, & \overline{e_{a}^{\mu}}=e_{a}^{\bar{\mu}}=e_{R_{a}^{\mu}}^{\mu}-i e_{I_{a}^{\check{\mu}}}^{\check{\mu}} \\
e_{\mu}^{a}=e_{R}^{a}{ }_{\mu}-i e_{I_{\breve{\mu}}^{a}}^{a}, & \overline{e_{\mu}^{a}}=e_{\bar{\mu}}^{a}=e_{R}^{a}+i e_{I_{\breve{\mu}}^{a}}^{a} .
\end{array}
$$

Vielbeins are holomorphic functions, and thus transform as holomorphic vectors (we consider only the transformation of the Greek indices for this purpose),

$$
\begin{aligned}
& e_{\mu}^{a}\left(z^{\nu}\right) \rightarrow \tilde{e}_{\mu}^{a}\left(w^{\nu}\right)=\frac{\partial z^{\alpha}\left(w^{\nu}\right)}{\partial w^{\mu}} e_{\alpha}^{a}\left(z^{\rho}\right) \\
& e_{a}^{\mu}\left(z^{\nu}\right) \rightarrow \tilde{e}_{b}^{\mu}\left(w^{\nu}\right)=\frac{\partial w^{\mu}\left(z^{\nu}\right)}{\partial z^{\alpha}} e_{b}^{\alpha}\left(z^{\rho}\right)
\end{aligned}
$$

The holomorphy of vielbeins,

$$
\frac{\partial}{\partial z^{\bar{\mu}}} e_{\nu}^{a}=\frac{1}{2}\left[\frac{\partial}{\partial x^{\mu}}+i \frac{\partial}{\partial y^{\check{\mu}}}\right]\left[e_{R}^{a}+i e_{I_{\breve{v}}^{a}}^{a}\right]=0,
$$

\footnotetext{
${ }^{5}$ Here the Latin indices $a, b$ run from $0,1, \ldots, d-1$, since they represent local indices, and $\eta_{\mu \nu}=$ $\operatorname{diag}(-1,1,1,1)$.

${ }^{6}$ Checks $(\checkmark)$ are put on indices to denote the imaginary part of a coordinate or on indices of objects, which are projected onto their basis vectors.
} 
implies the Cauchy-Riemann equations,

$$
\frac{\partial e_{R}^{a}}{\partial x^{\mu}}=\frac{\partial e_{I_{\check{\nu}}}^{a}}{\partial y^{\check{\mu}}}, \quad \frac{\partial e_{I_{\check{\nu}}^{a}}^{a}}{\partial x^{\mu}}=-\frac{\partial e_{R}^{a}}{\partial y^{\check{\mu}}} .
$$

When rotating the metric from $z$ and $\bar{z}$ space to $x$ and $y$ space, the components of the holomorphic rotated metric $\boldsymbol{g}_{m n}$ are given by

$$
\boldsymbol{g}_{m n}=\left(\begin{array}{ll}
g_{\mu \nu} & g_{\mu \check{\nu}} \\
g_{\breve{\mu} \nu} & g_{\breve{\mu} \check{\nu}}
\end{array}\right)=\frac{1}{2}\left(\begin{array}{cc}
C_{\mu \nu}+C_{\bar{\mu} \bar{\nu}} & i\left(C_{\mu \nu}-C_{\bar{\mu} \bar{\nu}}\right) \\
i\left(C_{\mu \nu}-C_{\bar{\mu} \bar{\nu}}\right) & -C_{\mu \nu}-C_{\bar{\mu} \bar{\nu}}
\end{array}\right) .
$$

Its inverse then becomes

$$
\boldsymbol{g}^{m n}=\frac{1}{2}\left(\begin{array}{cc}
C^{\mu \nu}+C^{\bar{\mu} \bar{\nu}} & i\left(-C^{\mu \nu}+C^{\bar{\mu} \bar{\nu}}\right) \\
i\left(-C^{\mu \nu}+C^{\bar{\mu} \bar{\nu}}\right) & -C^{\mu \nu}-C^{\bar{\mu} \bar{\nu}}
\end{array}\right) .
$$

Clearly the entries of these rotated metrics are all symmetric and real. It is easily verified that $C_{\mu \nu}=g_{\mu \nu}-i K_{\mu \nu}$ and that $C_{\bar{\mu} \bar{\nu}}=g_{\mu \nu}+i K_{\mu \nu}, K_{\mu \nu}=g_{\mu \check{\nu}}=g_{\check{\mu}}$ is a symmetric metric tensor and $g_{\breve{\mu} \check{\nu}}=-g_{\mu \nu}$. We can write the metric in terms of the real and imaginary parts of the vielbein and in terms of the imaginary part of $C_{\mu \nu}$, using the definition of the complex metric in terms of vielbeins (12), yields

$$
\boldsymbol{g}_{m n}=\left(\begin{array}{cc}
e_{\mu} e_{\nu}-e_{\breve{\mu}} e_{\breve{v}} & -e_{\breve{\mu}} e_{\nu}-e_{\mu} e_{\breve{v}} \\
-e_{\breve{\mu}} e_{\nu}-e_{\mu} e_{\breve{v}} & -e_{\mu} e_{\nu}+e_{\breve{\mu}} e_{\breve{v}}
\end{array}\right)
$$

Expressing the inverse metric $\boldsymbol{g}^{m n}$ in terms of vielbeins yields

$$
\left(\begin{array}{cc}
g^{\mu \nu} & K^{\mu \nu} \\
K^{\mu \nu} & -g^{\mu \nu}
\end{array}\right)=\left(\begin{array}{cc}
e^{\mu} e^{\nu}-e^{\check{\mu}} e^{\check{\nu}} & e^{\check{\mu}} e^{\nu}+e^{\mu} e^{\check{\nu}} \\
e^{\check{\mu}} e^{\nu}+e^{\mu} e^{\check{\nu}} & -e^{\mu} e^{\nu}+e^{\check{\mu}} e^{\check{\nu}}
\end{array}\right) .
$$

With the rotated metric we can write down the holomorphic line element in its rotated form

$$
d s^{2}=g_{\mu \nu} d x^{\mu} d x^{\nu}-\frac{2 G_{N}}{c^{3}} K_{\mu \nu} d p^{\mu} d x^{\nu}-\frac{G_{N}^{2}}{c^{6}} g_{\mu \nu} d p^{\mu} d p^{\nu} .
$$

From the definition of the complex metric in terms of vielbeins (12) and the fact that the vielbeins are defined to satisfy

$$
e_{a}^{\mu} e_{v}^{a}=\delta_{v}^{\mu},
$$

it follows that $\boldsymbol{g}^{m e}$ and $\boldsymbol{g}_{\text {en }}$ are inverses of each other

$$
\boldsymbol{g}^{m e} \boldsymbol{g}_{e n}=\boldsymbol{\delta}_{n}^{m},
$$

where $\delta_{n}^{m}$ is defined as

$$
\delta_{n}^{m} \equiv\left(\begin{array}{cc}
\delta_{v}^{\mu} & 0 \\
0 & \delta_{\bar{v}}^{\bar{\mu}}
\end{array}\right)
$$




\section{Flat Space}

The holomorphic line element (19) in flat space becomes

$$
d s^{2}=-(c d t)^{2}+(d \vec{x})^{2}-\left[-\left(d y^{0}\right)^{2}+(d \vec{y})^{2}\right] .
$$

Note that, although it may seem that this metric is invariant under the action of the $S O(4,4)$, as already pointed out in Sect. 2 it is in fact invariant under the $S O(1,3 ; \mathbb{C})$. Indeed, the complexified Lorentz group is (by definition) the group which leaves the holomorphic line element (9) invariant, and the invariance is not changed by rotating to the $x, y$ coordinates, where the $y$ coordinate is related to the momentum-energy coordinate $[1,17]$ (see also Sect. 10) by $y^{\mu} \equiv p^{\mu} G_{N} / c^{3}$. The space-time-momentumenergy interval squared from the origin to a space-time-momentum-energy point $\boldsymbol{x}^{m}$ is given by

$$
d^{2}\left(\mathbf{0} ; \boldsymbol{x}^{m}\right)=-(c t)^{2}+(\vec{x})^{2}-\frac{G_{N}^{2}}{c^{6}}\left[-\left(\frac{E}{c}\right)^{2}+(\vec{p})^{2}\right] .
$$

Note that the contribution of momentum-energy to the line element multiplies $G_{N}^{2} / c^{6}$, which is tiny $\left(G_{N}^{2} / c^{6} \sim 10^{-72} \mathrm{~s}^{2} / \mathrm{kg}^{2}\right)$, as it should be, since one does not observe any momentum-energy contribution to (21) at low energies. For light-like propagation $\left(d s^{2}=0\right)$ and in the absence of momentum-energy contribution, (21) reduces to the well known result: massless particles move on the light-cone with the speed of light, $v=\|d \vec{x} / d t\|=c$.

On the other hand, for a light-like propagation in the presence of a non-vanishing momentum-energy contributions however, (21) yields,

$$
-(c t)^{2}+(\vec{x})^{2}-\frac{p^{2} G_{N}^{2}}{c^{6}}=0,
$$

where the variable $p^{2}=\eta_{\mu \nu} p^{\mu} p^{\nu}$ is the momentum-energy squared in a space-timemomentum-energy hyper-surface. Setting the space-time-momentum-energy interval to zero determines the boundary of causality (light cones). We can specify our hypersurface further by setting $t$ to zero. Assuming $p^{2}>0$, one finds that there is a spatial region which is in instantaneous causal contact, and whose radius is given by,

$$
r_{\max }=\frac{G_{N} \sqrt{p^{2}}}{c^{3}} \quad\left(p^{2}>0\right) .
$$

The causally related regions (time-like, $d s^{2}<0$ ) for the case $p^{2}>0$ are shown as white in Fig. 1, where the region of non-local causal contact at $t=0$ is clearly seen as the hyperbola's throat. On the other hand, when $p^{2}<0$, the causal boundaries, shown in Fig. 2, are quite different. There is a minimal time $t_{\min }$ required for events 
Fig. 1 The figure shows how the light cones get modified by non inertial coordinate transformations on a space-time-momentum-energy diagram in the case when $p^{2}>0$. Note that there is a non-local instantaneous causally related volume element near the origin

Fig. 2 The figure shows how the light cones get modified by non-inertial coordinate transformations when $p^{2}<0$. There is a minimum time interval required for events to be in causal contact

to be in causal contact,

$$
t_{\min }=\frac{G_{N} \sqrt{-p^{2}}}{c^{4}} \quad\left(p^{2}<0\right) \text {. }
$$

ct
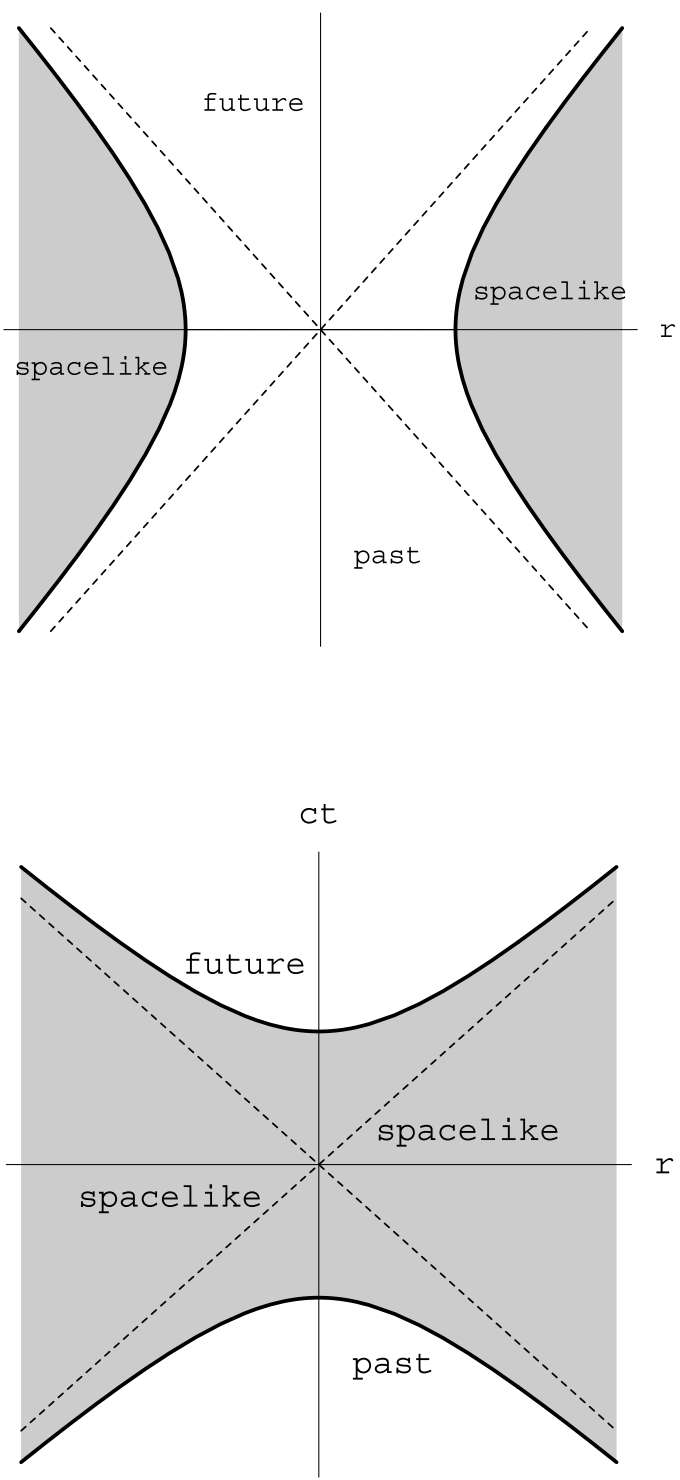
We now calculate the phase velocity, using the space-time-momentum-energy line element (22)

$$
v_{\text {phase }}=\frac{x}{t}=\sqrt{c^{2}+\frac{p^{2} G_{N}^{2}}{c^{4} t^{2}}} .
$$

One can see that the phase velocity approaches the speed of light for large $t$.

On the other hand, the group velocity becomes

$$
v_{\mathrm{g}}=\sqrt{c^{2}+\frac{G_{N}^{2}}{c^{6}} f^{2}},
$$

where

$$
f^{2}=-\frac{1}{c^{2}}\left(\frac{d E}{d t}\right)^{2}+\left(\frac{d \vec{p}}{d t}\right)^{2}
$$

The group velocity approaches the speed of light for small four-forces squared. For small four-forces the group velocity (24) can be expanded as

$$
v_{g} \simeq c\left[1+3.4 \times 10^{-89} \mathrm{~kg}^{-2} \mathrm{~m}^{-2} \mathrm{~s}^{4} f^{2}\right] .
$$

From this we see that the four-forces need to be immense to cause any significant deviation of $v_{\mathrm{g}}$ from $c$. For example, for $f^{2} \simeq \eta_{\mu \nu} f^{\mu} f^{v}$ of the order of $f^{2} \simeq\left(10^{38} N\right)^{2}$ we get a deviation $\left|v_{g}-c\right| \simeq 10^{-4} \mathrm{~m} / \mathrm{s}$. This deviation is comparable with the current experimental bounds [18] (see also Refs. [19, 20] which report more stringent tests). By making use of a laser version of the Kennedy-Thorndyke experiment (an improved version of the Michelson-Morley experiment originally designed to measure the effects of the luminiferous aether through which the Earth presumably moves), Hils and Hall have tested the universality of the speed of light in free space $c$ up to an accuracy of $6 \times 10^{-5} \mathrm{~m} / \mathrm{s}$, comparable to the deviation mentioned above. Unless one faces conditions of extremely large four-forces, holomorphic gravity describes the speed of light in free space extremely well. Such strong four-forces could be present only in strong gravitational fields, e.g. close to the black hole and Big Bang singularities. However, general relativity in strong gravitational fields has up to now not been tested. It would be of interest do design a thought experiment, in which physical processes in a strong gravitational field could have an observational impact on an asymptotic observer placed in a weak field. Such candidates could be accretion disks of large rotating black holes in the presence of strong magnetic fields, which are present for example in the vicinity of active galactic nuclei.

Requiring that $v_{g}$ in (24) be real and positive (as required by propagation) sets a lower limit on the four force, $f^{2} \geq-c^{8} / G_{N}^{2}$. This is to be contrasted with an upper limit occurring in Hermitian gravity [1]. Just like in the case of Hermitian gravity, we expect that these apparent violations of causality become of importance only in the regions where the particle's self-gravity is large (in the vicinity of particle's own event horizon). 


\section{Second Order Formalism}

Let us now state the holomorphic Einstein-Hilbert action, ${ }^{7}$

$$
S=\frac{c^{4}}{16 \pi G_{N}} \int d^{8} z \sqrt{-C} R+\text { c.c. }
$$

The holomorphic Ricci scalar is defined by $R=C^{\mu \nu} R_{\mu \nu}$, where the holomorphic Ricci tensor is given by

$$
R_{\mu \lambda \nu}^{\lambda}=\partial_{\lambda} \Gamma_{v \mu}^{\lambda}-\partial_{\nu} \Gamma_{\lambda \mu}^{\lambda}+\Gamma_{\lambda \alpha}^{\lambda} \Gamma_{v \mu}^{\alpha}-\Gamma_{v \alpha}^{\lambda} \Gamma_{\lambda \mu}^{\alpha},
$$

and where here $\partial_{\lambda}=\partial / \partial z^{\lambda}$. Since the holomorphic Einstein-Hilbert action is just the complexified equivalent of the Einstein-Hilbert action of standard general relativity, the equations of motions for this theory are just complexified equivalents of the equations of motions of general relativity. Using first order formalism [21], the equations of motion implied by the action (26) are:

$$
\begin{aligned}
G_{\mu \nu} & =\frac{8 \pi G_{N}}{c^{4}} T_{\mu \nu}, \\
\nabla_{\rho} C^{\mu \nu} & =0 .
\end{aligned}
$$

Through the holomorphic metric compatibility equations (28b) the holomorphic connection coefficients are given by

$$
\Gamma_{\mu \nu}^{\rho}=\frac{1}{2} C^{\rho \epsilon}\left(\partial_{\mu} C_{\epsilon \nu}+\partial_{\nu} C_{\mu \epsilon}-\partial_{\epsilon} C_{\mu \nu}\right) .
$$

Plugging the holomorphic Levi-Cività connection coefficients into the holomorphic Einstein's equations (28a), we obtain second order differential equations in terms of the holomorphic metric only.

When varying the single particle action $S=-m \int d s+$ h.c. where $d s$ is the holomorphic line element (11) we obtain the following holomorphic geodesic equations

$$
\ddot{z}^{\rho}+\Gamma_{\mu \nu}^{\rho} \dot{z}^{\mu} \dot{z}^{v}=0
$$

and its complex conjugate. We can write equations (30) in their convenient eight dimensional form, such that we can rotate them to $x, y$ space, by simply plugging in the rotated metric (18). This is possible because the holomorphic connection coefficients transform as a $(1,2)$ tensor under the constant coordinate transformations such as rotations from $z, \bar{z}$ to $x, y$ space. The rotated eight dimensional connection coefficients are then simply given by

$$
\boldsymbol{\Gamma}_{m n}^{r}=\frac{1}{2} \boldsymbol{g}^{r e}\left(\partial_{m} \boldsymbol{g}_{e n}+\partial_{n} \boldsymbol{g}_{m e}-\partial_{e} \boldsymbol{g}_{m n}\right)
$$

\footnotetext{
${ }^{7}$ In contrast to Hermitian gravity [1], the holomorphy symmetry of the metric tensor of holomorphic gravity can be imposed both on- and off-shell (at the level of the action). This has the advantage that the action (26) suffices to fully specify the dynamics of holomorphic gravity, and no further (action) constraints are needed. Furthermore, it can be easily shown that the Bianchi identities and the covariant stress-energy conservation are satisfied.
} 
where the metric $\boldsymbol{g}_{m n}$ is just the eight dimensional rotated metric (16).

At a first sight, the geodesic equation (30) is a rather strange generalization of general relativity, in that it requires twice as many initial conditions to fully specify the geodesic motion of a particle. We shall now provide a plausible physical meaning for all initial conditions, and explain how they reduce to the standard initial conditions of general relativity. In holomorphic gravity there are in total 16 initial conditions, namely:

$$
\begin{aligned}
& \left(x^{\mu}\right)_{0} ; \quad\left(u^{\mu}\right)_{0}=\left(\frac{d x^{\mu}}{d \tau}\right)_{0} \\
& \left(p^{\mu}\right)_{0} ; \quad\left(f^{\mu}\right)_{0}=\left(\frac{d p^{\mu}}{d \tau}\right)_{0} .
\end{aligned}
$$

These conditions reduce to the initial conditions of general relativity when the following on-shell condition (see also Sect. 10) is satisfied

$$
p^{\mu}=m u^{\mu} \quad \text { (on-shell), }
$$

where $m$ denotes particle's mass, but we emphasise that the initial conditions (32) do not require or imply (33). When particle's mass vanishes, the on-shell condition becomes $v=c\|\vec{k}\|$, with $p^{0}=\hbar v / c, p^{i}=\hbar k^{i}$, where $\hbar$ denotes the reduced Planck constant, $v$ particle's frequency and $\vec{k}$ its wavenumber. From (33) and the geodesic equation (30) we also know the 4-force

$$
f^{\mu}=\frac{d p^{\mu}}{d \tau}=m \frac{d u^{\mu}}{d \tau}=-m \Re\left[\Gamma_{\alpha \beta}^{\mu} \dot{z}^{\alpha} \dot{z}^{\beta}\right] \quad \text { (on-shell), }
$$

where $\dot{z}^{\alpha}=u^{\alpha}+i\left(G_{N} / c^{3}\right) f^{\alpha}$. This gives an algebraic equation for $f^{\mu}$. Together with (33), this equation completely defines the on-shell initial conditions.

However, in general neither (33) nor (34) is fulfilled. Just like for a quantum particle (recall the phase space representation of a coherent state), in holomorphic gravity there is no direct relation between particle's momentum and the rate of change of its position (velocity), and thus to fully specify particle's motion in holomorphic gravity, one needs all of the initial conditions (32).

Complete ramifications of these considerations can be appreciated only within a quantum theory of gravity, which shall be considered in a future publication within the context of a hermitian theory of gravity ( $c f$. Ref. [1]).

\section{The Limit to General Relativity}

The limit of Holomorphic gravity to general relativity is based on the assumption that the $y$ coordinate and its corresponding vielbein are small. When expanding the equations of holomorphic gravity in powers of $y$ and its corresponding vielbein, we hope to obtain the theory of general relativity at zeroth order of the expansion and 
meaningful corrections at linear order. The easiest way to obtain the limit to general relativity is to expand the vielbeins in terms of the $y$ coordinate

$$
e_{\mu}^{a}(x, y)=\mathrm{e}^{i y \cdot \partial_{x}} e_{\mu}^{a}(x) .
$$

From this and the definition, $C_{\mu \nu}=e_{\mu}(z) \cdot e_{\nu}(z)$, we conclude that the analogous formula holds for the holomorphic metric tensor, $C_{\mu \nu}(x, y)=\mathrm{e}^{i y \cdot \partial_{x}} C_{\mu \nu}(x)$, such that,

$$
\begin{aligned}
& g_{\mu \nu}(x, y)=\cos \left(y \cdot \partial_{x}\right) g_{\mu \nu}(x)+\sin \left(y \cdot \partial_{x}\right) K_{\mu \nu}(x) \\
& K_{\mu \nu}(x, y)=-\sin \left(y \cdot \partial_{x}\right) g_{\mu \nu}(x)+\cos \left(y \cdot \partial_{x}\right) K_{\mu \nu}(x),
\end{aligned}
$$

where we made use of (16) and (18). The analogous relations hold for the inverses $g^{\mu \nu}$ and $K^{\mu \nu}$. Assuming that $K_{\mu \nu}$ is-just like $y^{\mu}$-a first order quantity, (36) then tells us that $g_{\mu \nu}(x, y)$ acquires corrections at second order in $y$, while $K_{\mu \nu}(x, y)$ acquires first order corrections in $y$, with $K_{\mu \nu}$ being on its own a first order quantity.

This analysis then implies that the connection with all indices unchecked yields the ordinary Levi-Cività connection plus corrections of second order

$$
\Gamma_{\mu \nu}^{\rho}(x, y)=\Gamma_{\mu \nu}^{\rho}(x)+O\left(y^{2}\right) .
$$

With these connection coefficients, we can know check if the theory reduces to the theory of general relativity by plugging them into the rotated eight dimensional geodesic equation. Keeping only terms of linear order in the $y$ coordinate and its corresponding vielbein, yields the ordinary geodesic equation

$$
\ddot{x}^{\rho}+\Gamma_{\mu \nu}^{\rho}(x) \dot{x}^{\mu} \dot{x}^{\nu}+O\left(y^{2}\right)=0
$$

without any first order corrections present. We have not studied in detail the reduction of holomorphic Einstein's equations to general relativity. Our study of holomorphic Schwarzschild solution and cosmology in Sects. 7 and 9 suggests however that holomorphic gravity reduces to Einstein's theory in the low energy limit.

\subsection{Scalar Field}

In this subsection we consider the following scalar field action

$$
S_{\phi}=\int d^{8} z \sqrt{-C}\left[-\frac{1}{2} C^{\mu \nu}\left(\nabla_{\mu} \phi\right) \nabla_{\nu} \phi-V(\phi)\right]+\text { c.c. },
$$

where $\phi=\phi\left(z^{\mu}\right)$ is a holomorphic function of $z^{\mu}$. The equations of motion for the scalar field are then

$$
\square \phi-\frac{d V}{d \phi}=0
$$

and its complex conjugate, where $\square=C^{\mu \nu} \nabla_{\mu} \nabla_{\nu}$ and $\bar{\square}=C^{\bar{\mu} \bar{\nu}} \nabla_{\bar{\mu}} \nabla_{\bar{\nu}}$. When varying this action with respect to the metric we obtain the two sets of components of the 
stress-energy tensor

$$
T_{\alpha \beta}=\nabla_{\alpha} \phi \nabla_{\beta} \phi-\frac{1}{2} C_{\alpha \beta} C^{\mu \nu} \nabla_{\mu} \phi \nabla_{\nu} \phi-C_{\alpha \beta} V(\phi)
$$

and its complex conjugate. The energy conservation equations are

$$
\nabla_{\mu} T^{\mu \nu}=\left(\square \phi-\frac{d V}{d \phi}\right) \nabla^{v} \phi=0
$$

and its complex conjugate, where we have used (38). There are two sets of Einstein's equations, namely

$$
R_{\mu \nu}=\frac{8 \pi G_{N}}{c^{4}}\left(\nabla_{\mu} \phi \nabla_{\nu} \phi+C_{\mu \nu} V(\phi)\right)
$$

and again its complex conjugate. The energy-momentum tensor of a perfect fluid in the fluid rest frame can be written in the diagonal form

$$
T_{v}^{\mu}=\operatorname{diag}(-\rho, p, p, p)
$$

plus its complex conjugate, where $\rho$ is the density and $p$ the pressure. For a homogeneous scalar field $\phi=\phi(t)$, (39) yields, $\rho=\left(1 / 2 c^{2}\right) \dot{\phi}^{2}+V(\phi)$ and $p=$ $\left(1 / 2 c^{2}\right) \dot{\phi}^{2}-V(\phi)$.

\section{The Holomorphic Schwarzschild Solution}

The holomorphic Schwarzschild solution should just be a complexified version of the ordinary Schwarzschild solution, since the holomorphic Einstein's equations (28a) are just complexified versions of the ordinary Einstein's equations. So we expect to have the holomorphic metric components

$$
\begin{gathered}
C_{00}=-\left(1-\frac{1}{c^{2}} \frac{2 G_{N} M}{z}\right), \\
C_{11}=\left(1-\frac{1}{c^{2}} \frac{2 G_{N} M}{z}\right)^{-1}
\end{gathered}
$$

and their complex conjugates, where $z=\|\vec{z}\|=\|\vec{x}\|+i \frac{G_{N}}{c^{3}}\|\vec{p}\| \equiv r+i \frac{G_{N}}{c^{3}} p$ and $M$ is the mass of the black hole, which in holomorphic gravity could, in principle, be complex. ${ }^{8}$ The angular part takes the same form as in general relativity

$$
d \Omega^{2}=d \theta^{2}+\sin ^{2} \theta d \phi^{2}
$$

\footnotetext{
${ }^{8}$ At this moment we do not have a good physical interpretation for $\Im[M]$. In this paper we take $M$ to be real.
} 
but now the spherical symmetry is $O(3 ; \mathbb{C})$ and thus the angular parameters are complex

$$
\theta=\theta_{R}+i \theta_{I} \quad \phi=\phi_{R}+i \phi_{I}
$$

Thus the angular part of the holomorphic Schwarzschild solutions is the complex angular part (44) plus its complex conjugate. One can easily check that the holomorphic Schwarzschild solution is indeed a solution to the holomorphic Einstein's equations (28a) in vacuüm. Expressing the Schwarzschild components (43) in $r$ and $p$ yields

$$
\begin{aligned}
& C_{00}=-\left(1-\frac{2 G_{N} M}{c^{2}} \frac{r-i \frac{G_{N}}{c^{3}} p}{r^{2}+\frac{G_{N}^{2}}{c^{6}} p^{2}}\right) \\
& C_{11}=\frac{r^{2}+\frac{G_{N}^{2}}{c^{6}} p^{2}-\frac{2 G_{N} M}{c^{2}}\left(r+i \frac{G_{N}}{c^{3}} p\right)}{\left(r-\frac{2 G_{N} M}{c^{2}}\right)^{2}+\frac{G_{N}^{2}}{c^{6}} p^{2}}=-\frac{1}{C_{00}}
\end{aligned}
$$

and their complex conjugates. The rotated holomorphic metric components (16) are given in terms of the holomorphic metric components (45), and take the following form

$$
\begin{aligned}
& g_{00}=-\left(1-\frac{2 G_{N} M}{c^{2}} \frac{r}{r^{2}+\frac{G_{N}^{2}}{c^{6}} p^{2}}\right), \\
& g_{11}=\frac{r^{2}+\frac{G_{N}^{2}}{c^{6}} p^{2}-\frac{2 G_{N} M}{c^{2}} r}{\left(r-\frac{2 G_{N} M}{c^{2}}\right)^{2}+\frac{G_{N}^{2}}{c^{6}} p^{2}}, \\
& g_{0 \check{0}}=\frac{\frac{2 G_{N}^{2} M}{c^{5}} p}{r^{2}+\frac{G_{N}^{2}}{c^{6}} p^{2}}, \\
& g_{1 \check{1}}=\frac{\frac{2 G_{N}^{2} M}{c^{5}} p}{\left(r-\frac{2 G_{N} M}{c^{2}}\right)^{2}+\frac{G_{N}^{2}}{c^{6}} p^{2}},
\end{aligned}
$$

which reduces to the Schwarzschild solution of general relativity when $p \rightarrow 0$. When these rotated components are inserted into the rotated holomorphic line element (19), one obtains the holomorphic Schwarzschild metric in its full glory

$$
\begin{aligned}
d s^{2}= & -\left(1-\frac{2 G_{N} M}{c^{2}} \frac{r}{r^{2}+\frac{G_{N}^{2}}{c^{6}} p^{2}}\right)\left[(c d t)^{2}-\frac{G_{N}^{2}}{c^{8}}(d E)^{2}\right] \\
& +\frac{r^{2}+\frac{G_{N}^{2}}{c^{6}} p^{2}-\frac{2 G_{N} M}{c^{2}} r}{\left(r-\frac{2 G_{N} M}{c^{2}}\right)^{2}+\frac{G_{N}^{2}}{c^{6}} p^{2}}\left[(d r)^{2}-\frac{G_{N}^{2}}{c^{6}}(d p)^{2}\right]
\end{aligned}
$$




$$
\begin{aligned}
& +\frac{\frac{2 G_{N}^{2} M}{c^{5}} p}{\left(r-\frac{2 G_{N} M}{c^{2}}\right)^{2}+\frac{G_{N}^{2}}{c^{6}} p^{2}} \frac{2 G_{N}}{c^{3}} d r d p \\
& +\frac{\frac{2 G_{N}^{2} M}{c^{5}} p}{r^{2}+\frac{G_{N}^{2}}{c^{6}} p^{2}} \frac{2 G_{N}}{c^{4}} d t d E+\left(r^{2}-\frac{G_{N}^{2}}{c^{6}} p^{2}\right)\left(d \theta_{R}^{2}-d \theta_{I}^{2}\right) \\
& -4 \frac{G_{N}}{c^{3}} r p d \theta_{R} d \theta_{I}+\left(d \phi_{R}^{2}-d \phi_{I}^{2}\right) \\
& \times\left[\left(r^{2}-\frac{G_{N}^{2}}{c^{6}} p^{2}\right)\left\{\left(\sin \theta_{R} \cosh \theta_{I}\right)^{2}-\left(\cos \theta_{R} \sinh \theta_{I}\right)^{2}\right\}\right. \\
& \left.-4 \frac{G_{N}}{c^{3}} r p\left(\sin \theta_{R} \cosh \theta_{I} \cos \theta_{R} \sinh \theta_{I}\right)\right] \\
& -2 d \phi_{R} d \phi_{I}\left[\left(r^{2}-\frac{G_{N}^{2}}{c^{6}} p^{2}\right) 2 \sin \theta_{R} \cosh \theta_{I} \cos \theta_{R} \sinh \theta_{I}\right. \\
& \left.+2 \frac{G_{N}}{c^{3}} r p\left\{\left(\sin \theta_{R} \cosh \theta_{I}\right)^{2}-\left(\cos \theta_{R} \sinh \theta_{I}\right)^{2}\right\}\right] .
\end{aligned}
$$

This rotated holomorphic Schwarzschild metric is indeed a solution of the rotated holomorphic Einstein's equations in vacuüm, as can be checked by explicit calculation of $R_{\mu \nu}$. It is easy to see that, in the limit when the radius $r$ goes to infinity and $p$ to zero, the solution approaches the Minkowski metric, whereas the solution approaches the momentum-energy Minkowski space when $r$ goes to zero and $p$ goes to infinity.

\subsection{Curvature Singularities}

If $p$ is not zero when $r$ is zero, there is no curvature singularity at the origin. Explicit calculation shows that $p$ is not zero when $r$ is zero for a generic infalling observer. When considering an observer which is falling in radially we can neglect the change in angular coordinates, corresponding to a vanishing angular momentum. (Recall that in general relativity this choice corresponds to the worst case scenario, for which an observer descends in the quickest possible way towards the black hole singularity.) Based on the holomorphic Schwarzschild metric (43) the black hole line element (10) of a radially infalling observer can be recast as

$$
\begin{aligned}
-2 c^{2}= & -\left(1-\frac{1}{c^{2}} \frac{2 G_{N} M}{z}\right)\left(\frac{d z^{0}}{d \tau}\right)^{2} \\
& +\left(1-\frac{1}{c^{2}} \frac{2 G_{N} M}{z}\right)^{-1}\left(\frac{d z}{d \tau}\right)^{2}+\text { c.c. }
\end{aligned}
$$

where we introduced a real affine parameter (proper time) $\tau$ defined as $c^{2}(d \tau)^{2}=$ $-d s^{2}$. The holomorphic Schwarzschild solution has an isometry in the $z^{0}$ direction 
$\left(\partial / \partial z^{0}\right.$ is a Killing vector) and hence there is a conserved quantity:

$$
e_{z}=u_{0}^{0}+i \frac{G_{N}}{c^{3}} f_{0}^{0}
$$

The real part $u_{0}^{0}=u^{0}\left(\tau_{0}\right)$ corresponds to the (initial) zeroth component of the fourvelocity evaluated at $\tau=\tau_{0}$ (the energy per unit mass divided by $c$ ), which is also the Killing vector in general relativity, while the imaginary part $f_{0}^{0}=f^{0}\left(\tau_{0}\right)=$ $\left(d p^{0} / d \tau\right)\left(\tau_{0}\right)$ is the zeroth component of the observer's four-force. Since $f_{0}^{0}$ does not contribute in general relativity, the general relativistic limit should be obtained by setting $f_{0}^{0} \rightarrow 0$, which is indeed the case. In analogy with general relativity, the conserved quantity can be written as [3]

$$
\left(\frac{d z^{0}}{d \tau}\right)^{2}=\left(1-\frac{1}{c^{2}} \frac{2 G_{N} M}{z}\right)^{-2} e_{z}^{2}
$$

Equations (47) and (49) are not enough to fully determine $z=z(\tau)$, because nothing is known about the imaginary part of the expressions appearing in (47). Since we are in holomorphic gravity, it is reasonable to demand $z=z(\tau)$ to be a holomorphic function. With this analytic extension we can now completely determine $z=z(\tau)$ by inserting the conserved integral (49) into (47), to obtain

$$
\left(\frac{d z}{c d \tau}\right)^{2}=b+\frac{r_{s}}{z}, \quad b=\frac{e_{z}^{2}}{c^{2}}-1, \quad r_{s}=\frac{2 G_{N} M}{c^{2}}
$$

and the complex conjugate of this equation. Note that $r_{s}$ is just the Schwarzschild radius and the on-shell value of $b=b_{r}+i b_{i}$ can be expressed in terms of the initial 3-velocity, $b_{r}=\gamma_{0}^{2}-1=\left(\gamma_{0} \vec{v}_{0} / c\right)^{2}, \gamma_{0}^{-2}=1-\left(\vec{v}_{0} / c\right)^{2}$. Solving (50) for proper time $\tau$ yields

$$
\begin{aligned}
\frac{c \tau}{r_{s}}= & \frac{1}{b} \sqrt{\zeta(1+b \zeta)} \\
& -\frac{1}{b^{\frac{3}{2}}} \ln \{\sqrt{b \zeta}+\sqrt{1+b \zeta}\}, \quad \zeta=\frac{z}{r_{s}} .
\end{aligned}
$$

From this it follows that, when $b$ is complex and $r \rightarrow 0, p$ is not zero. Moreover, one can show that quite generically, when $r \rightarrow 0, p$ grows large, which limits the growth of the curvature invariant (2), which for Holomorphic gravity has the simple generalization,

$$
R_{\mu \nu \rho \sigma} R^{\mu \nu \rho \sigma}=\frac{12 r_{s}^{2}}{z^{6}}
$$

For example, when $r \rightarrow 0$, this reduces to $R_{\mu \nu \rho \sigma} R^{\mu \nu \rho \sigma} \rightarrow-12 c^{18} r_{s}^{2} /\left(G_{N} p\right)^{6}$, which is negative.

In order to verify the limited curvature conjecture, we solve (50) numerically. For initial conditions, where the imaginary part of $b$ is nonvanishing, $p$ is indeed nonzero when $r=0$, as can be seen from Fig. 3(a). In this case there is no curvature 
Fig. 3 The real and imaginary part and the absolute value of the complex radial coordinate $z$ as a function of proper time $\tau$ for a freely falling observer for different initial conditions $b$.

(a) The real and imaginary part and the absolute value of $z$ with a complex initial condition $b$. In this case $|z|=0$ is never reached, indicating that the observer encounters no black hole singularity. (b) The real and imaginary part and the absolute value of $z$ for a real initial $b$. In this case both $\mathfrak{R}[z]$ and $\Im[z]$ reach simultaneously zero for some value of proper time $\tau$, indicating a black hole singularity.

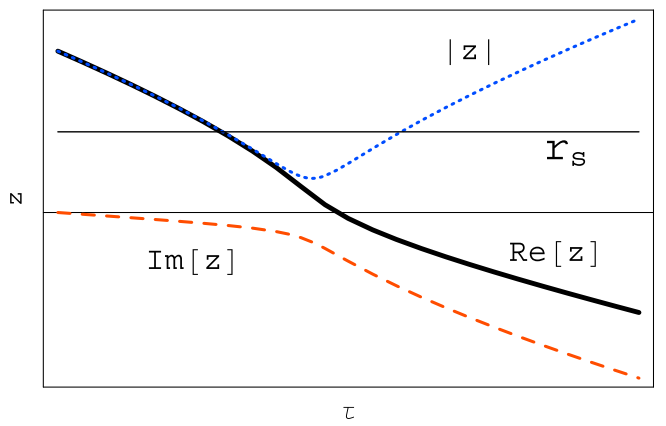

(a)

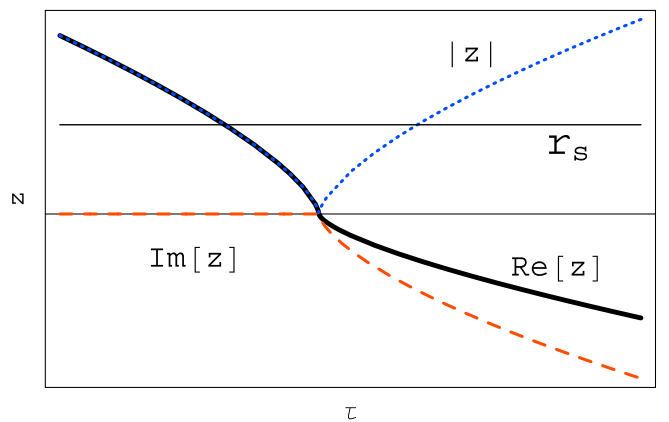

(b)

singularity. When $\Im[b]=0$ however, then $r$ and $p$ simultaneously go to zero, the geodesics end at a curvature singularity, just like in the case of general relativity. This situation is illustrated in Fig. 3(b). Even though numerical solution indicates that the evolution continues after $r=0=p$ is reached, at that point there is a branch point of the evolution, and the numerical integrator picks one of the Riemann sheets (this is indicated by the cuspy feature of the numerical solution at $r=p=0$ in Fig. 3(b)). Moreover, at $r=0=p$ the curvature invariant (51) becomes singular, as can be seen in Fig. 5. Since $\Im[b]=0$ only when the initial force $f_{0}^{0}=0$ exactly, the set of initial conditions where $b$ is real is of measure zero, when compared to the set of all initial conditions, where $b$ can be an arbitrary complex number. Hence we conclude that the curvature singularity is not seen by most of infalling observers. The above observations are made within the coordinate choice, which maximize the Kretchmann invariant (51) when $r=0$ and $p=0$.

Finally, we need to interpret what it means for $r$ to become negative, see Fig. 3. In the view of (50), for instance, we can take the sign out of the denominator and put it in the numerator

$$
\frac{M}{-r+i\left(G_{N} / c^{3}\right) p}=\frac{-M}{r-i\left(G_{N} / c^{3}\right) p} .
$$

In this way we effectively glue the holomorphic Schwarzschild solution with a negative $\Re[z]$ onto the anti-holomorphic solution with a positive $\Re[z]$ but with a negative mass: a white hole. This means that an observer can fall through a black hole 


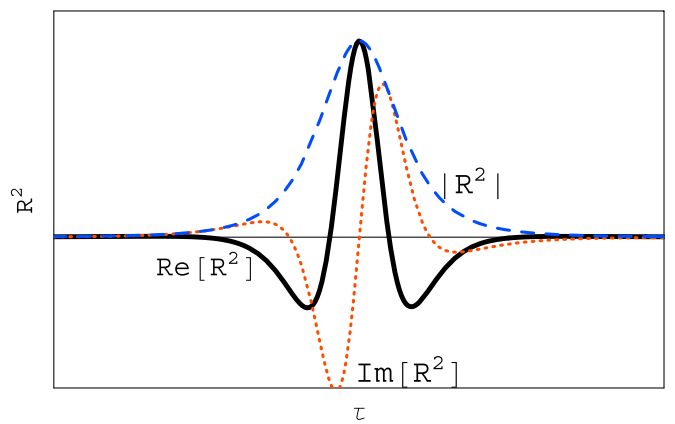

(a)

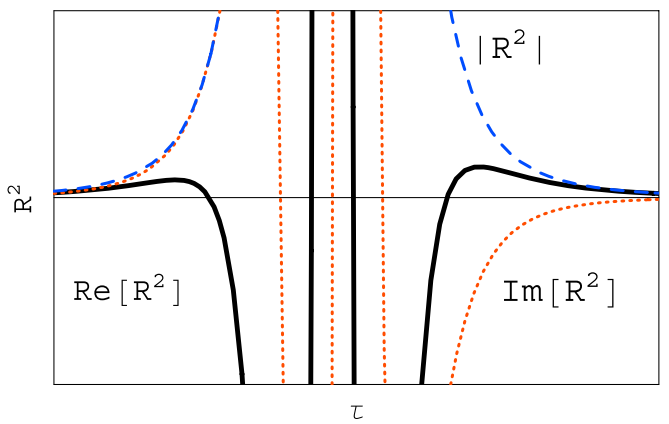

(b)

Fig. 4 The real and imaginary part and the absolute value of the curvature invariant (51), which we for simplicity denote in figures by $R^{2}$. as a function of proper time $\tau$ for different initial conditions $b$. An infinite curvature is attained only when $\Im[b]=0$, which represents a set of initial conditions of measure zero. (a) The real and imaginary part and the absolute value of the curvature invariant for complex initial conditions $b$. The curvature invariant remains finite at all times. (b) A part of the upper panel (a) zoomed in. At large distances the real part of the curvature invariant (solid black line) approaches the general relativistic solution, but close to and within the Schwarzschild radius the curvature exhibits a very different behavior, becoming even negative. The deviation from the general relativistic behavior becomes large only within the Schwarzschild radius and hence remains hidden behind the black hole horizon.

and emerge from a white hole with an opposite momentum and v.v. Needless to say, this behavior is very different from general relativity, where classically nothing can come out once inside the event horizon. Finally, we note that the holomorphic and anti-holomorphic solutions are in general not symmetric under time reversal. This symmetry is broken, for example, by the imaginary part of the curvature invariant, as can be seen in Fig. 4(a).

\subsection{The Weak Field limit and Light Deflection}

The weak field (Newtonian) limit of general relativity tells us that the Newtonian potential $\phi_{N}$ is related to the metric as follows

$$
g_{00}=-\left(1+\frac{2 \phi}{c^{2}}\right)
$$


Fig. 5 The real and imaginary part and the absolute value of the curvature invariant (51) with initial conditions corresponding to a real $b$ (vanishing initial force). Since $\Im[z]=0$ remains zero, the curvature invariant is identical to the corresponding curvature invariant in general relativity (1) such that a curvature singularity is reached at $r=0=p$

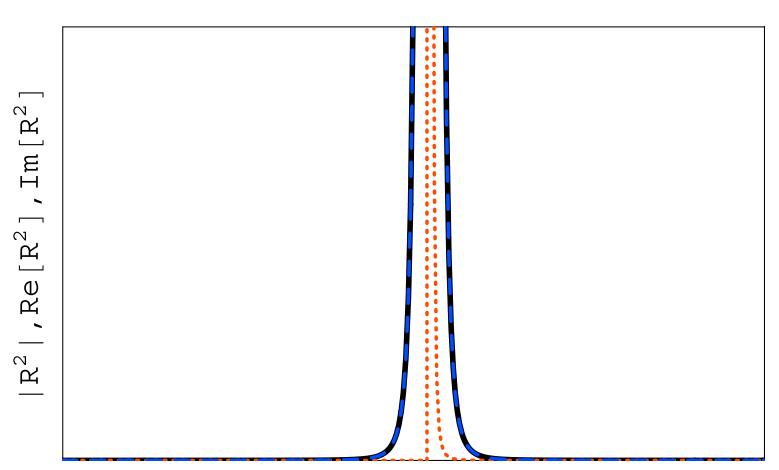

$\tau$

Solving for the Newtonian potential, using the expression for the metric component (46a), yields the standard Newtonian potential plus a small correction

$$
\phi=-G_{N} M \frac{r}{r^{2}+\frac{G_{N}^{2}}{c^{2}} p^{2}} \simeq-\frac{G_{N} M}{r}\left[1-\frac{G_{N}^{2} p^{2}}{c^{6} r^{2}}\right] .
$$

That this correction is indeed small can be seen by rewriting the relative correction in (54) as,

$$
\frac{G_{N}^{2} p^{2}}{c^{6} r^{2}}=\frac{p^{2}}{p_{\mathrm{P}}^{2}} \frac{\ell_{\mathrm{P}}^{2}}{r^{2}}
$$

where $p_{\mathrm{P}}=\sqrt{\hbar c^{3} / G_{N}} \simeq 1.2 \times 10^{19} \mathrm{GeV} / c$ and $\ell_{\mathrm{P}}=\sqrt{\hbar G_{N} / c^{3}} \simeq 1.6 \times 10^{-35} \mathrm{~m}$ denote the Planck momentum and the Planck length, respectively. Thus for a particle of Planck energy the 'post-Newtonian' correction is significant only up to distances of the order the Planck length, hence unobservably small.

If we would like to know how light gets deflected around a massive object, it is necessary to consider holomorphic Maxwell fields. The action is in eight dimensional notation given by

$$
S_{\mathrm{M}}=-\int d^{8} \boldsymbol{z}\left(\frac{1}{4} \boldsymbol{C}^{m n} \boldsymbol{C}^{m^{\prime} n^{\prime}} \boldsymbol{F}_{m m^{\prime}} \boldsymbol{F}_{n n^{\prime}}+\frac{e}{c^{2}} \boldsymbol{C}^{m n} \boldsymbol{A}_{m} \boldsymbol{J}_{n}\right),
$$

which is in four dimensional notation given by

$$
S_{\mathrm{M}}=-\int d^{8} z\left(\frac{1}{4 c} C^{\mu v} C^{\mu^{\prime} \nu^{\prime}} F_{\mu \mu^{\prime}} F_{\nu v^{\prime}}+\frac{e}{c^{2}} C^{\mu v} A_{\mu} J_{v}+\text { c.c. }\right),
$$

where $e$ and $J_{v}=J_{v}\left(z^{\alpha}\right)$ denote the electromagnetic current and electric charge, respectively. The holomorphic Maxwell field strength is given by

$$
F_{\mu \nu}=\nabla_{\mu} A_{\nu}-\nabla_{\nu} A_{\mu}=\partial_{\mu} A_{\nu}-\partial_{\nu} A_{\mu} .
$$


Notice that the components of the vector field $A^{\mu}$ are holomorphic functions $A_{\mu}=A_{\mu}\left(z^{\gamma}\right)$. Varying the holomorphic Maxwell action yields the equation of motion

$$
\nabla_{\mu} \nabla^{\mu} A^{v}-\nabla^{v} \nabla_{\mu} A^{\mu}-R_{\mu}^{v} A^{\mu}=\frac{e}{c} J^{v}
$$

To get the last two terms on the l.h.s. we made use of

$$
\nabla_{\mu} \nabla^{v} A^{\mu}=\nabla^{v} \nabla_{\mu} A^{\mu}+R_{\mu}^{v} A^{\mu}
$$

which is obtained by contracting the standard identity, $\left[\nabla_{\mu}, \nabla_{\nu}\right] A_{\rho}=R_{\rho \nu \mu}^{\sigma} A_{\sigma}$.

Working in a covariant Lorenz gauge $\nabla_{\mu} A^{\mu}=0$, (56) become

$$
\square A^{v}-R_{\mu}^{v} A^{\mu}=\frac{e}{c} J^{v}, \quad \nabla_{\mu} A^{\mu}=0,
$$

where $\square=C^{\mu \nu} \nabla_{\mu} \nabla_{\nu}$ denotes the holomorphic d'Alembertian operator. Since in the holomorphic Schwarzschild space-time the Ricci tensor vanishes, $R_{\mu}^{v}=0$, (57) implies that the polarization of light does not affect its propagation. This shows that, just like in general relativity, light deflection in the Schwarzschild holomorphic spacetime can be studied via the geodesic equation for massless point-like particles (30), which is what we do next.

To linear order in the (holomorphic) potential $\Phi=\Phi(\vec{z})$ the holomorphic metric (cf. (43)) reads

$$
C_{\mu \nu}=\eta_{\mu \nu}+\frac{2}{c^{2}} \Phi \delta_{\mu \nu}+\mathcal{O}\left(\Phi^{2}\right)
$$

where $\delta_{\mu \nu}=\eta_{\mu \nu}+2 \delta_{\mu}^{0} \delta_{v}^{0}$ is the unity matrix (Kronecker delta). When the potential is sourced by a static point particle of a mass $M$, then (43))

$$
\Phi=-\frac{G_{N} M}{z}
$$

Similarly the Levi-Cività connection (29) reads,

$$
\Gamma_{\mu \nu}^{\alpha}=\frac{\eta^{\alpha \beta}}{c^{2}}\left(\delta_{\beta \nu} \partial_{\mu}+\delta_{\mu \beta} \partial_{\nu}-\delta_{\mu \nu} \partial_{\beta}\right) \Phi+\mathcal{O}\left(\Phi^{2}\right),
$$

whose nonvanishing components are,

$$
\begin{aligned}
& \Gamma_{00}^{i}=\Gamma_{i 0}^{0}=\Gamma_{0 i}^{0}=-\frac{1}{c^{2}} \partial_{i} \Phi \\
& \Gamma_{i j}^{k}=\frac{1}{c^{2}}\left(\delta_{i}^{k} \partial_{j}+\delta_{j}^{k} \partial_{i}-\delta_{i j} \partial_{k}\right) \Phi .
\end{aligned}
$$

For a photon the zeroth order geodesic equation (in the absence of gravitational field) tells us simply that the photon is moving with the speed of light, $u^{0}=\|\vec{u}\|=c$ and that the velocity four vector $u^{\mu(0)}$ is null, $\eta_{\mu \nu} u^{\mu(0)} u^{\nu(0)}=0$. Here $u^{0}=d z^{0^{(0)}} / d \tau$ 
and $u^{i}=d z^{i(0)} / d \tau$ and $u^{\mu}=u^{\mu(0)}+\delta u^{\mu}$. From (30) and (59) we easily get the geodesic equations for $\delta u^{\mu}$ accurate to the first order in $\Phi$,

$$
\frac{d \delta u^{0}}{d \tau} \simeq \frac{2}{c} \vec{u}^{(0)} \cdot \nabla \Phi, \quad \frac{d \delta \vec{u}}{d \tau} \simeq 2 \nabla_{\perp} \Phi,
$$

where $\nabla_{\perp}=\nabla-\vec{u}^{(0)}\left(\vec{u}^{(0)} \cdot \nabla\right)$ denotes the holomorphic derivative in the direction orthogonal to the photon motion. Introducing a proper length $\ell=c \tau$, the second equation in (60) can be integrated once to get $\delta \vec{u}=(2 / c) \int d \ell \nabla_{\perp} \Phi$. Dividing this by the speed of propagation $c$ along the (complex) path $\ell$, one gets the holomorphic deflection angle,

$$
\vec{\alpha}_{h}=\frac{2}{c^{2}} \int d \ell \nabla_{\perp} \Phi
$$

Just like in the example where we solved the geodesic equation for a particle freely falling onto a black hole, we have analytically extended the solution to the geodesic equation $\delta \vec{u}(\ell)$ by complexifying the path $\ell$. As an example we now solve (61) for the potential of a static point like particle (58) to obtain

$$
\alpha_{h}=-\frac{4 G_{N} M}{c^{2} z_{1}},
$$

where $z_{1}$ denotes the closest 'distance' (impact parameter) to the lens located at $\vec{z}=0$. In evaluating the integral in (61) we have, for simplicity, assumed that both the observer and the photon source are very distant from the lense. The physical deflection angle is obtained by rotating (62). The result is

$$
\alpha=-\frac{4 G_{N} M x_{1}}{c^{2} x_{1}^{2}+G_{N}^{2} p_{1}^{2} / c^{4}} \simeq-\frac{4 G_{N} M}{c^{2} x_{1}}\left(1-\frac{G_{N}^{2} p_{1}^{2}}{c^{6} x_{1}^{2}}\right) .
$$

Comparing this with (55) we see that the correction to $\alpha$ induced by holomorphic gravity is indeed unobservably small. We have thus shown that, up to a small relative correction of the form (55), holomorphic gravity reproduces correctly both the Newton law for slow massive bodies and the Einstein's light deflection formula.

\section{Gravitational Waves}

Holomorphic gravity is very similar to general relativity. This is also the case for gravitational waves, since holomorphic gravity has a similar structure of degrees of freedom, except that it hosts complex instead of real degrees of freedom. Hence here we present the main steps in the derivation, which is carried through by similar reasoning as in general relativity. We consider weak gravitational fields, which allow us to decompose the holomorphic metric into the flat Minkowsi metric plus a small perturbation.

$$
C_{\mu \nu}=\eta_{\mu \nu}+h_{\mu \nu}
$$


where the metric perturbation is a holomorphic function, which under infinitezimal coordinate transformations, $x^{\gamma} \rightarrow x^{\gamma}+\xi^{\gamma}$, transforms as follows

$$
h_{\mu \nu} \rightarrow h_{\mu \nu}+\partial_{\mu} \xi_{v}+\partial_{\nu} \xi_{\mu},
$$

where $\xi_{\mu}=\xi_{\mu}\left(z^{\gamma}\right)$ are holomorphic functions. To linear order in $h_{\mu \nu}$ the connection coefficients read,

$$
\Gamma_{\mu \nu}^{\rho}=\frac{1}{2} \eta^{\rho \lambda}\left(\partial_{\mu} h_{\nu \lambda}+\partial_{\nu} h_{\lambda \mu}-\partial_{\lambda} h_{\mu \nu}\right)
$$

With these connections coefficients the linearized vacuum Einstein's equations become

$$
\begin{aligned}
G_{\mu \nu}= & \frac{1}{2}\left(\partial_{\sigma} \partial_{\nu} h_{\mu}^{\sigma}+\partial_{\sigma} \partial_{\mu} h_{\nu}^{\sigma}-\partial_{\mu} \partial_{\nu} h-\square h_{\mu \nu}\right. \\
& \left.-\eta_{\mu \nu} \partial_{\rho} \partial_{\lambda} h^{\rho \lambda}+\eta_{\mu \nu} \square h\right)=0,
\end{aligned}
$$

where $\square=\eta^{\rho \sigma} \partial_{\rho} \partial_{\sigma}$.

We consider transverse traceless gauge, which can be fixed by constraining $\xi_{v}$ in (65) as follows [22],

$$
\begin{aligned}
& \vec{\partial}^{2} \vec{\xi}+\frac{1}{3} \vec{\partial}(\vec{\partial} \cdot \vec{\xi})=0, \\
& \vec{\partial}^{2} \xi_{0}-\partial_{0}(\vec{\partial} \cdot \vec{\xi})=0 .
\end{aligned}
$$

These gauge contraints imply

$$
\begin{aligned}
& \vec{\nabla} \cdot s=0 \quad s_{i j}=\frac{1}{2} h_{i j}-\frac{1}{6} \eta^{k l} h_{k l} \eta_{i j} \\
& \vec{\nabla} \cdot \vec{w}=0, \quad w_{i}=h_{0 i},
\end{aligned}
$$

as can be checked by using the transformation of the metric perturbation (65). Upon fixing the transverse gauge and demanding well behaved boundary conditions and taking into account holomorphicity of the metric perturbations, we are left with the (transverse, traceless) $i j$ components of Einsteins equations (68), which can be written as the wave equation,

$$
\square h_{\mu \nu}^{T T}=0,
$$

where $h_{\mu 0}^{T T}=0, h_{i j}^{T T}=2 s_{i j}$. From (69) we see that $h_{\mu \nu}^{T T}$ contains two complex degrees of freedom, which are the physical degrees of freedom, and which as usually can be represented by the + and $\times$ polarizations.

The general solution of (71) in the wave zone (in which gravitational waves propagate radially and respect spherical symmetry) can be written in terms of the outward (right, R) and inward (left, L) moving waves as follows,

$$
h_{\mu \nu}^{T T}\left(z^{0}, z\right)=\sum_{\alpha=+, \times} h_{\mu \nu}^{\alpha, R}\left(z^{0}-z\right)+h_{\mu \nu}^{\alpha, L}\left(z^{0}+z\right),
$$


where $z=\|\vec{z}\|$ and $h_{\mu \nu}^{\alpha, R}$ and $h_{\mu \nu}^{\alpha, L}$ are (arbitrary) holomorphic functions of its argument. Notice that, because of holomorphy, the number of physical gravitational degrees of freedom (two) is the same in holomorphic gravity as in general relativity. The observed wave is, as usually, obtained by rotating (72) (cf. (16)),

$$
\left.h_{\mu \nu}^{T T}\right|_{\mathrm{obs}}=\frac{1}{2}\left(h_{\mu \nu}^{T T}\left(z^{0}, z\right)+h_{\bar{\mu} \bar{\nu}}^{T T}\left(z^{\overline{0}}, \bar{z}\right)\right) .
$$

Because of the holomorphy symmetry the off-diagonal components of the metric tensor $K_{\mu \nu}$ can be obtained as the analytic extension of $\left.h_{\mu \nu}^{T T}\right|_{\text {obs }}$ and hence do not contain any new physical information. Moreover, as can be seen from (19), $K_{\mu \nu}$ couples to $\left(G_{N} / c^{3}\right) d p^{\mu} d x^{\nu}$, implying that the contribution of $K_{\mu \nu}$ to distance measurements (as performed by gravitational wave observatories) is suppressed by $G_{N} / c^{3}$, and thus negligibly small for small energies and momenta. Furthermore, in the low energy regime, where (36) applies, (72) can be expanded in powers of $p^{\mu}$ as,

$$
\begin{aligned}
\left.h_{\mu \nu}^{T T}\right|_{\mathrm{obs}}= & \frac{1}{2}\left(h_{\mu \nu}^{T T}\left(x^{0}, r\right)+h_{\bar{\mu} \frac{T}{\nu}}^{T}\left(x^{0}, r\right)\right) \\
& +\mathcal{O}\left(\frac{G_{N} E}{c^{4}}, \frac{G_{N} p}{c^{3}}\right), \quad\left(r=\|\vec{x}\|, x^{0}=c t\right),
\end{aligned}
$$

which, to leading order in $G_{N} E / c^{4}$ and $G_{N} p / c^{3}$, agrees with general relativity. Indeed, typical gravitational waves from astrophysical sources have wavelengths larger than a meter, such that $\left\|p^{\mu}\right\| \ll\left(c^{3} / G_{N}\right)\left\|\partial_{\mu}\right\|^{-1}$, where $\left\|\partial_{\mu}\right\|^{-1}>1 \mathrm{~m}$ denotes the length scale over which $h_{\mu \nu}^{T T}$ varies significantly.

A gravitational wave moving in $x^{3}$ direction with a definite polarization (say $h^{+}$in (72)) will result in an invariant (observable) change in the distance $\Delta L_{1}=$ $\int_{0}^{L_{0}}\left(d s-d s_{0}\right)$ along the $x^{1}$ direction, where $d s_{0}$ denotes the unperturbed (Minkowski) line element. Integrating $d s$ in (10) and keeping only the linear terms in $h^{+} \equiv h_{11}^{+}$ one obtains,

$$
\Delta L_{1}=L_{0} \frac{1}{2} \Re\left[h^{+}\right]-\frac{G_{N}}{c^{3}} p^{1} \Im\left[h^{+}\right],
$$

where $p^{1}$ denotes the space-time momentum. As discussed above, it is reasonable to take this to be the on-shell momentum of particles in the wave, $p^{1}=\hbar k^{1}$, where $k^{1}$ is the wave vector in the $x^{1}$ direction. Since the typical gravitational wave vectors are $k=2 \pi / \lambda$, with $\lambda$ greater than about a meter (corresponding to the shortest wavelength measured by earthly experiments) the latter contribution in (75) for a meter wave is of the order $G_{N} h /\left(c^{3} \lambda\right) \sim 10^{-69} \mathrm{~m}$, which, when multiplied by $\Im\left[h^{+}\right] \ll 1$, results in an unobservably small correction to the first term in (75).

It is also of interest to consider the energy carried by the gravitational wave (72). Analytic extension of the well known formula from general relativity suggests that the (complex) Hamiltonian density corresponding to gravitational waves on flat space is,

$$
\mathcal{H}_{\mathrm{gw}}=\frac{1}{64 \pi G_{N}}\left[\dot{h}_{\mu \nu}^{T T} \dot{h}^{\mu \nu T T}+\sum_{i=1}^{3}\left(\partial_{z^{i}} h_{\mu \nu}^{T T}\right)\left(\partial_{z^{i}} h^{\mu \nu T T}\right)\right] .
$$


This result can be also obtained by varying the quadratic action in small perturbations $h_{\mu \nu}=C_{\mu \nu}-C_{\mu \nu}^{\mathrm{b}}$ with respect to the background metric $C_{\mu \nu}^{\mathrm{b}}$, and then setting $C_{\mu \nu}^{\mathrm{b}}=\eta_{\mu \nu}$. The (observable) energy density carried by gravitational waves corresponds to the real part of (76):

$$
\rho_{\mathrm{gw}}=\Re\left[\mathcal{H}_{\mathrm{gw}}\right]=\frac{1}{32 \pi G_{N}} \sum_{\alpha=+, \times}\left[\left(\dot{h}^{\alpha, R}\right)_{r}^{2}-\left(\dot{h}^{\alpha, R}\right)_{i}^{2}\right],
$$

where we dropped the left moving wave in (72) and we made use of,

$$
h_{\mu \nu}^{T T}=\sum_{\alpha=+, \times} \epsilon_{\mu \nu}^{\alpha}\left(h_{r}^{\alpha, R}+\imath h_{i}^{\alpha, R}\right), \quad \epsilon_{\mu \nu}^{\alpha} \epsilon^{\mu \nu \beta}=\delta^{\alpha \beta} .
$$

Now since the imaginary contribution to the metric sourcing gravitational waves is typically suppressed by $\left(G_{N} / c^{3}\right) p / r$ with respect to the real part of the metric (see, for example, (45)), we expect that the same suppression carries over to the generated gravitational waves. Hence, for sub-Planckian energies we expect $\left\|\dot{h}_{r}^{\alpha}\right\| \gg\left\|\dot{h}_{i}^{\alpha}\right\|$, implying the positivity of energy (77) carried by gravitational waves, and thus the absence of ghosts. One might be tempted to conclude that the negative sign of the second term in (77) indicates that term can grow in magnitude without limits, leading to an energy unbounded from below, and thus ruling out the theory in question. Theories with such a behaviour are said to contain a ghost field. Ghost fields are present in certain complexified theories of general relativity [23, 24], making them thus unacceptable on physical grounds. Yet there is no ghost in holomorphic gravity simply because holomorphy is a stronger principle than the principle of energy minimization. ${ }^{9}$ An alternative way of resolving the ghost problem has been proposed by extending general relativity to pseudocomplex spaces $[25,26]$.

\section{Cosmology}

Since our Universe is isotropic and homogeneous on large scales, our complex generalized theory should also possess isotropy and homogeneity such that it reduces to the theory of general relativity correctly at low energies. Since our complex theories are completely specified in terms of vielbeins, an assumption concerning the vielbein modeling an isotropic and homogeneous universe is in place here. Let us try the following Ansatz:

$$
e_{\mu}^{a}(z)=a\left(z_{c}^{0}\right) \delta_{\mu}^{a} \quad e_{\bar{\mu}}^{a}(\bar{z})=\bar{a}\left(z_{c}^{\overline{0}}\right) \delta_{\bar{\mu}}^{a},
$$

where

$$
a\left(z_{c}^{0}\right)=a_{R}\left(z_{c}^{0}\right)+i a_{I}\left(z_{c}^{0}\right) \quad \bar{a}\left(z_{c}^{\overline{0}}\right)=a_{R}\left(z_{c}^{\overline{0}}\right)-i a_{I}\left(z_{c}^{\overline{0}}\right)
$$

\footnotetext{
${ }^{9}$ Indeed, when applied to the gravitational wave amplitudes (78), the Cauchy-Riemann equations $\partial_{y} \mu h_{i}^{\alpha}=$ $\partial_{x} \mu h_{r}^{\alpha}$ and $\partial_{x} \mu h_{i}^{\alpha}=-\partial_{y} \mu h_{r}^{\alpha}$ tell us that $h_{i}^{\alpha}$ is completely specified (up to an irrelevant constant) in terms of $h_{r}^{\alpha}$.
} 
where $z_{c}^{0}=\eta+i G_{N} E_{c}$ denotes a conformal complex 'time.' (In this section we set the speed of light $c=1$.) With these assumptions the holomorphic Christoffel symbols (29) are then given by

$$
\Gamma_{\mu \nu}^{\rho}=\frac{a^{\prime}}{a}\left(\delta_{\nu}^{0} \delta_{\mu}^{\rho}+\delta_{\mu}^{0} \delta_{\nu}^{\rho}-\eta^{\rho 0} \eta_{\mu \nu}\right)
$$

and its complex conjugate, where $a^{\prime}=d a / d z_{c}^{0}$. Inserting (80) into the holomorphic Riemann tensor (27) we obtain

$$
\begin{aligned}
R_{\mu \nu}= & {\left[\frac{a^{\prime \prime}}{a}-\left(\frac{a^{\prime}}{a}\right)^{2}\right]\left(\eta_{\mu \nu}-2 \delta_{\nu}^{0} \delta_{\mu}^{0}\right) } \\
& +\left(\frac{a^{\prime}}{a}\right)^{2}\left[2 \delta_{\nu}^{0} \delta_{\mu}^{0}+2 \eta_{\mu \nu}\right]
\end{aligned}
$$

and its complex conjugate. The holomorphic Ricci scalars are then given by

$$
R=6 \frac{a^{\prime \prime}}{a^{3}}=6\left[\frac{\ddot{a}}{a}-\left(\frac{\dot{a}}{a}\right)^{2}\right]
$$

and its complex conjugate, where we have contracted holomorphic Ricci tensors with the inverse metric $g^{\mu \nu}=\left(1 / a^{2}\right) \eta^{\mu \nu}$ and its complex conjugate, and $\dot{a}=d a / d z^{0}$, $d z^{0}=a d z_{c}^{0}$. The holomorphic Einstein's equations in four dimensions are

$$
R_{\mu \nu}=8 \pi G_{N}\left(T_{\mu \nu}-\frac{1}{2} g_{\mu \nu} T\right)
$$

and its complex conjugate, of course. Because of the isotropy and homogeneity symmetries imposed, the Einstein's equations contain only four independent equations due to homogeneity and isotropy symmetries, namely one space-space (ii) equation,

$$
\frac{\ddot{a}}{a}+2\left(\frac{\dot{a}}{a}\right)^{2}=4 \pi G(\rho-p),
$$

the time-time (00) equation,

$$
\frac{\ddot{a}}{a}=-\frac{4 \pi G}{3}(\rho+3 p)
$$

and the complex conjugates of these equations. We can simplify (81) by making use of the time component (82) to obtain

$$
H^{2} \equiv\left(\frac{\dot{a}}{a}\right)^{2}=\frac{8 \pi G_{N}}{3} \rho,
$$

where $H=\dot{a} / a$ denotes the holomorphic Hubble parameter. Equation (83) together with (82) constitute the holomorphic Friedmann equations. It is easy to see that the 
holomorphic Friedmann equations are consistent with the holomorphic energy conservation equations

$$
\dot{\rho}+3 \frac{\dot{a}}{a}(\rho+p)=0
$$

and its complex conjugate, which is derived from the holomorphic energy conservation equations

$$
\partial_{\mu} T_{0}^{\mu}+\Gamma_{\mu \lambda}^{\mu} T_{0}^{\lambda}-\Gamma_{\mu 0}^{\lambda} T_{\lambda}^{\mu}=0
$$

and its complex conjugate, where we have used the expression of the energymomentum tensor (41) and holomorphic Christoffel symbol (80). Making use of (41) and (39), we can express the energy density $\rho$ and pressure $p$ in terms of the scalar field $\phi$. In an isotropic and homogeneous universe the scalar field is a holomorphic function of $z^{0}, \phi=\phi\left(z^{0}\right)$, such that we have

$$
\rho=\frac{1}{2} \dot{\phi}^{2}+V(\phi), \quad p=\frac{1}{2} \dot{\phi}^{2}-V(\phi) .
$$

The equations for $\bar{\rho}$ and $\bar{p}$ are just the complex conjugates of these expressions. The equation of motion for the scalar field (38) becomes

$$
\ddot{\phi}+3 H \dot{\phi}+\frac{d V}{d \phi}=0
$$

and the holomorphic Friedmann equations (82-83) then become

$$
\frac{\ddot{a}}{a}=-\frac{8 \pi G_{N}}{3}\left(\dot{\phi}^{2}-V\right)
$$

and

$$
H^{2} \equiv\left(\frac{\dot{a}}{a}\right)^{2}=\frac{8 \pi G_{N}}{3}\left(\frac{\dot{\phi}^{2}}{2}+V\right)
$$

and the corresponding complex conjugates. Just like in general relativity, one can show that only three equations among (84) and (86-88) are independent.

We shall now study the holomorphic Friedmann equations for different physical circumstances and compare the results with those of general relativity.

\subsection{Power Law Expansion}

Let us now consider a homogeneous and holomorphic fluid with the equation of state,

$$
w=\frac{p}{\rho}, \quad w \in C, \quad \Re[w] \geq-1 .
$$

In this case (84) is solved by,

$$
\rho=\frac{\rho_{0}}{a^{3(1+w)}} .
$$


Next (87) and (88) can be combined into,

$$
\epsilon \equiv-\frac{\dot{H}}{H^{2}}=\frac{d}{d z^{0}}\left(\frac{1}{H}\right)=\frac{3}{2}(1+w),
$$

such that $\epsilon \in \boldsymbol{C}$ is a complex constant and $\Re[\epsilon] \geq 0$.

When (91) is integrated, one gets the holomorphic expansion rate,

$$
H=\frac{1}{\epsilon z^{0}} .
$$

This is easily integrated, to yield the holomorphic scale factor,

$$
a=\left(\frac{z^{0}}{\zeta_{0}}\right)^{1 / \epsilon}
$$

These solutions are a generalization of power law expansion of general relativity, in that $\epsilon$ is in general a complex parameter. $\zeta_{0}$ is an arbitrary constant parameter that signifies the complex time at which $a=1$. Its physical significance is revealed by realizing that $\zeta_{0}$ parametrizes the Hubble rate at the time $z^{0}=\zeta_{0}$, as can be seen from (92) and (94).

Finally, the last equation we need to solve is (88), which gives,

$$
H_{0}^{2}=\frac{1}{\epsilon^{2} \zeta_{0}^{2}}=\frac{8 \pi G_{N} \rho_{0}}{3},
$$

where $H_{0}=H\left(\zeta_{0}\right), \rho_{0}=\rho\left(\zeta_{0}\right)$.

We shall now show that, non unlike in general relativity, the holomorphic power law expansion (93) can be realised by a homogeneous holomorphic scalar field $\phi=\phi\left(z^{0}\right)$ with an exponential potential. Just like in general relativity, the power law solution is then realised in the scaling limit, in which case it exhibits attractor behavior [27, 28].

Let us consider the following Lagrangian density

$$
\mathcal{L}=\frac{1}{2} \dot{\phi}^{2}-V(\phi), \quad V(\phi)=V_{0} \exp \left(-\lambda \frac{\phi}{M}\right)
$$

We now assume that the Friedmann equation permits a scaling solution of the form, $H \propto 1 / z^{0}$. Obviously, such a solution must satisfy,

$$
\dot{\phi}=\dot{\phi}_{0} \frac{\zeta_{0}}{z^{0}}, \quad \phi=\phi_{0}+\dot{\phi}_{0} \zeta_{0} \ln \left(\frac{z^{0}}{\zeta_{0}}\right),
$$

where $\phi_{0}, \dot{\phi}_{0}$ and $\zeta_{0}$ are (complex) constants (note that $\zeta_{0}$ and $\phi_{0}$ are not independent; indeed, rescaling $\zeta_{0}$ can be compensated by the appropriate shift in $\phi_{0}$ ). Note that (93) implies that the scalar field (96) can be considered as a 'marker' for the scale factor,

$$
a=\exp \left(\frac{\phi-\phi_{0}}{\epsilon \dot{\phi}_{0} \zeta_{0}}\right)
$$


and in this sense defines a clock.

A constant $w$ requires the scaling of the potential (95),

$$
V=V_{0} \exp \left(-\frac{\lambda \phi_{0}}{M}\right)\left(\frac{\zeta_{0}}{z^{0}}\right)^{\left(\lambda \dot{\phi}_{0} \zeta_{0}\right) / M}
$$

We are free to absorb $\phi_{0}$ in $V_{0}$ by redefining, $V_{0} \exp \left(-\lambda \phi_{0} / M\right) \rightarrow V_{0}$. Demanding the scaling,

$$
V=V_{0}\left(\frac{\zeta_{0}}{z^{0}}\right)^{2}
$$

implies the following condition on the initial field velocity,

$$
\dot{\phi}_{0}=\frac{2 M}{\lambda \zeta_{0}}
$$

where $V_{0}=V\left(\phi_{0}\right)=V\left(\phi\left(\zeta_{0}\right)\right) .^{10}$

Taking account of these scalings, the equation of state parameter $w$ becomes indeed constant,

$$
w=\frac{\eta_{0}-1}{\eta_{0}+1}, \quad \eta_{0}=\frac{\dot{\phi}_{0}^{2}}{2 V_{0}}=\frac{2 M^{2}}{\lambda^{2} V_{0} \zeta_{0}^{2}}
$$

and hence $\epsilon$ in (91) is also constant,

$$
\epsilon=\frac{d}{d z^{0}}\left(\frac{1}{H}\right)=\frac{3 \eta_{0}}{1+\eta_{0}} .
$$

The conservation equation is now trivially satisfied, implying the energy density scaling, $\rho=\rho_{0} / a^{2 \epsilon}=\rho_{0}\left(\zeta_{0} / z^{0}\right)^{2}$. Finally, the Friedmann equation (88) and (94) yields the constraint

$$
H_{0}^{2}=\frac{8 \pi G_{N} V_{0}}{3-\epsilon} .
$$

When this is combined with (102), one gets the following algebraic equation for $\epsilon$

$$
\epsilon(\epsilon-3)\left(\epsilon-\frac{\lambda^{2}}{16 \pi G_{N} M^{2}}\right)=0,
$$

which links the parameters of the scalar theory with $\epsilon$. Note that $\epsilon$ does not depend on the initial conditions on the field, but only on the coupling parameters of the potential. This should not surprise us since we are considering an attractor solution.

From (104) it follows that de Sitter space $(\epsilon=0)$ and kination $(\epsilon=3)$ are solutions and have a special significance. One can show that $\mathfrak{R}[\epsilon] \leq 3$, the limit is saturated when $\lambda^{2}=16 \pi G_{N} M^{2}$. For larger values of $\lambda$ there is no scaling solution:

\footnotetext{
${ }^{10}$ One can show that, even when the condition (100) is not met, quite generally $\dot{\phi}$ eventually approaches the attractor solution for which (100) holds.
} 
$V$ redshifts faster than the kinetic term, resulting in $\epsilon \rightarrow 3$. de Sitter space $\epsilon=0$ is also a solution of (104), which is realised when $\dot{\phi}_{0}=0$. This is indeed a stable solution, for which kinetic energy vanishes, and $V=V_{0} \equiv \Lambda_{0} /\left(8 \pi G_{N}\right)$, where $\Lambda_{0}$ denotes the equivalent cosmological constant, which in holomorphic gravity can be complex.

The nontrivial solution of (104) is

$$
\epsilon=\frac{\lambda^{2}}{16 \pi G_{N} M^{2}}, \quad 0 \leq \Re[\epsilon] \leq 3
$$

which corresponds to the attractor solution when the condition $0 \leq \Re[\epsilon] \leq 3$ in (105) is satisfied. Of course, the solution (105) is more general than the corresponding solution of general relativity in that $\lambda$ and hence also $\epsilon$ can be complex. To appreciate the significance of a complex $\epsilon$, let us assume that the Universe follows the attractor behavior with $\epsilon$ given by (105). In this case the exponential potential (95) can be written as, ${ }^{11}$

$$
V \stackrel{\text { attractor }}{\longrightarrow} V_{0} \exp \left(-\sqrt{16 \pi G_{N} \epsilon} \phi\right), \quad 0 \leq \Re[\epsilon] \leq 3 .
$$

This potential can be used to obtain both an accelerating universe (for which $\mathfrak{R}[\epsilon]<1$ ) and a decelerating universe (with $\mathfrak{R}[\epsilon]>1$ ). Thus with the appropriate choice of $\lambda$ all standard cases in cosmology can be reproduced: radiation era $(\epsilon=2)$; matter era $(\epsilon=3 / 2)$; kination $(\epsilon=3)$, which is realized in the limit when $\lambda \rightarrow 0$ and $V_{0} \rightarrow 0$; inflation $(0<\Re[\epsilon] \ll 1)$, etc.

The physical Hubble parameter $\mathcal{H}$ is given in terms of the real part of the holomorphic expansion rate $H$ as, $\mathcal{H}^{2}=\mathfrak{R}\left[H^{2}\right]$. For a real $\epsilon(92)$ implies,

$$
\Re\left[H^{2}\right]=\frac{t^{2}-G_{N}^{2} E^{2}}{\epsilon^{2}\left[t^{2}+G_{N}^{2} E^{2}\right]^{2}} .
$$

For a complex $\epsilon$ the expression is more complicated,

$$
\Re\left[H^{2}\right]=\frac{\left(\epsilon_{R}^{2}-\epsilon_{I}^{2}\right)\left[t^{2}-G_{N}^{2} E^{2}\right]-4 \epsilon_{R} \epsilon_{I} t G_{N} E}{|\epsilon|^{4}\left[t^{2}+G_{N}^{2} E^{2}\right]^{2}} .
$$

In order to find out whether the holomorphic Big Bang singularity is ever reached, we need to study geodesic equations, which shall give us a crucial information on whether a freely falling observer experiences the holomorphic singularity in (107108) (cf. Ref. [1]).

The physical scale factor $\mathcal{A}$ can be obtained as the real part of the holomorphic scale factor (93) squared,

$$
\mathcal{A}^{2}=\left(\frac{t^{2}+G_{N}^{2} E^{2}}{\left|\zeta_{0}\right|^{2}}\right)^{\frac{\epsilon_{R}}{|\epsilon|^{2}}-\frac{\epsilon_{I}}{|\epsilon|^{2}} \operatorname{Arctan}\left(\frac{G_{N} E}{|t|}\right)}
$$

\footnotetext{
${ }^{11}$ Note that the scalar potential of hermitian gravity discussed in Ref. [1] differs from (106).
} 


$$
\begin{aligned}
& \times \cos \left(\left[\frac{2 \epsilon_{I}}{|\epsilon|^{2}}+\frac{2 \epsilon_{R}}{|\epsilon|^{2}} \operatorname{Arctan}\left(\frac{G_{N} E}{|t|}\right)\right]\right. \\
& \left.\times \ln \left(\frac{t^{2}+G_{N}^{2} E^{2}}{\left|\zeta_{0}\right|^{2}}\right)\right),
\end{aligned}
$$

where $\epsilon=\epsilon_{R}+i \epsilon_{I}$. When $\epsilon_{I}=0$ and provided $E$ does not grow with time (which is reasonable), the Universe approaches the standard FLRW cosmology. When however $\epsilon_{I} \neq 0$, the Universe's scale factor develops oscillations, which can result in significant differences between holomorphic and FLRW cosmology even at late times. This is a disadvantage of holomorphic cosmology when compared with, for example, Hermitian cosmology developed in Ref. [1]. Note that, when $\epsilon$ develops an imaginary part, then the potential (106) violates charge-parity symmetry. In this case, as $\phi$ evolves, $\Re[V]$ oscillates and can be either positive or negative. When the potential is negative, the Universe can enter an anti-de Sitter-like phase. As a consequence, the physical scale factor $\mathcal{A}^{2}$ in (109) can be either positive or negative. To prevent a negative value for $\mathcal{A}^{2}$ one can add a constant to $V$ (or a cosmological term), which will keep $\mathcal{A}^{2}$ positive at all times. This type of behavior can have relevance for the Universe's dark energy.

\subsection{Geodesic Equation}

In order to better understand the behavior of the expansion rate and the corresponding scale factor, we shall now consider a freely falling observer in the contracting phase. To do that, we need to solve the corresponding geodesic equation, which in holomorphic gravity has formally the same form as the corresponding geodesic equation of general relativity discussed for example in Ref. [1]. Taking account of the Christoffel symbol (80), the geodesic equation and the line element can be written in conformal time as,

$$
\begin{aligned}
& \frac{d u_{c}^{\mu}}{d \tau}+\frac{a^{\prime}}{a}\left(2 u_{c}^{0} u_{c}^{\mu}-\frac{\delta_{0}^{\mu}}{a^{2}}\right)=0, \\
& a^{2} \eta_{\alpha \beta} u_{c}^{\alpha} u_{c}^{\beta}+\bar{a}^{2} \eta_{\alpha \beta} u_{c}^{\bar{\alpha}} u_{c}^{\bar{\beta}}=-2,
\end{aligned}
$$

where $\tau$ is the (real) proper time of a freely falling observer (in the frame in which all 3-velocities vanish): $(d s)^{2}=-2(d \tau)^{2}$, and

$$
u_{c}^{\mu}=\frac{d x_{c}^{\mu}}{d \tau}
$$

is the 4-velocity in conformal coordinates $x_{c}^{\mu}=\left(\eta, x_{c}^{i}\right)$. Defining the physical 4velocity as

$$
u^{\mu}=a u_{c}^{\mu}=a \frac{d x_{c}^{\mu}}{d \tau}
$$

we can rewrite the spatial and time component of the geodesic equation (110) as,

$$
\frac{d\left(a u^{i}\right)}{d \tau}=0, \quad \frac{d\left\{a^{2}\left[\left(u^{0}\right)^{2}-1\right]\right\}}{d \tau}=0 .
$$


These are solved by the following scaling solution,

$$
\frac{\left(u^{0}\right)^{2}-1}{\left(u_{0}^{0}\right)^{2}-1}=\frac{a_{0}^{2}}{a^{2}}, \quad \frac{u^{i}}{u_{0}^{i}}=\frac{a_{0}}{a},
$$

where $u_{0}^{\mu}=u^{\mu}\left(\tau_{0}\right)$ and $u^{\mu}=u^{\mu}(\tau)$. We introduce a complex constant $U$ by recasting the first equation in (114) as

$$
\begin{aligned}
u^{0} & \equiv \frac{d z^{0}}{d \tau}= \pm \sqrt{\frac{U}{a^{2}}+1} \\
U & \equiv a_{0}^{2}\left[\left(u_{0}^{0}\right)^{2}-1\right]=U_{R}+i U_{I} .
\end{aligned}
$$

When the line element (110) is taken account of, one would be tempted to identify,

$$
U=a_{0}^{2}\left\|\vec{u}_{0}\right\|^{2} .
$$

From the line element (110) it follows that, strictly speaking, only the real part of (115) must be satisfied. Yet by holomorphy (which we assume to dictate a unique analytic extension) we know that also the imaginary parts must match. We assume here that (115) indeed holds true.

Upon rewriting (115) in the integral form we get,

$$
\int \frac{d a a^{\epsilon}}{\sqrt{a^{2}+U}}=\frac{\tau}{\epsilon \zeta_{0}},
$$

where we made use of (93) and $d z^{0}=\epsilon z^{0} d a / a=\epsilon \zeta_{0} a^{\epsilon-1} d a$. Integrating (117) yields a hypergeometric function. Rather than performing a general analysis of (117), we shall consider the cosmologically interesting cases which are simple to analyze. We shall first consider a curvature dominated epoch with $\epsilon=1$.

\subsubsection{Curvature Dominated Epoch}

In this case (117) integrates to,

$$
\sqrt{a^{2}+U}=\frac{\tau}{\zeta_{0}}
$$

Note that is not a unique expression; there is a freedom to shift $\tau$ for an arbitrary real constant. From (118) it follows that,

$$
\begin{aligned}
a & =\sqrt{\left(\frac{\tau}{\zeta_{0}}\right)^{2}-U} \\
z^{0} & =\sqrt{\tau^{2}-U \zeta_{0}^{2}}=\sqrt{\tau^{2}-\zeta_{0}^{2}\|\vec{u}\|_{0}^{2}},
\end{aligned}
$$

from where we conclude,

$$
H^{2}=\frac{1}{\left(z^{0}\right)^{2}}=\frac{1}{\tau^{2}-\zeta_{0}^{2}\|\vec{u}\|_{0}^{2}}
$$


From (119) we get for the physical scale factor,

$$
\begin{aligned}
\mathcal{A}^{2} & =\Re\left[a^{2}\right]=\frac{\tau^{2}}{\left|\zeta_{0}\right|^{2}} \cos \left(2 \theta_{\zeta}\right)-|\vec{u}|_{0}^{2} \cos \left(2 \theta_{u}\right) \\
\|\vec{u}\|_{0} & =|\vec{u}|_{0} \mathrm{e}^{i \theta_{u}}, \quad \zeta_{0}=\left|\zeta_{0}\right| \mathrm{e}^{i \theta_{\zeta}},
\end{aligned}
$$

This then implies that the scale factor $\mathcal{A}^{2}$ will be positive at all times, provided both $\cos \left(2 \theta_{\zeta}\right)>0$ and $\cos \left(2 \theta_{u}\right)<0$, in which case the Big Bang singularity will never be reached. In order to have a more precise understanding on whether the Big Bang singularity is ever attained, we need to study a curvature invariant, one example being the Hubble parameter.

Observe that the physical Hubble parameter, which is obtained from (120),

$$
\mathcal{H}^{2}=\Re\left[H^{2}\right]=\frac{\tau^{2}-|\vec{u}|_{0}^{2}\left|\zeta_{0}\right|^{2} \cos (\vartheta)}{\left(\tau^{2}-|\vec{u}|_{0}^{2}\left|\zeta_{0}\right|^{2} \cos (\vartheta)\right)^{2}+|\vec{u}|_{0}^{4}\left|\zeta_{0}\right|^{4} \sin ^{2}(\vartheta)} .
$$

represents a bounce universe, where we defined $\vartheta=2\left(\theta_{u}+\theta_{\zeta}\right)$. When $\cos (\vartheta)>0$ the maximal physical expansion rate is reached when $\tau^{2}=|\vec{u}|_{0}^{2}\left|\zeta_{0}\right|^{2} \cos (\vartheta)+$ $|\vec{u}|_{0}^{2}\left|\zeta_{0}\right|^{2} \sin (\vartheta)$, for which

$$
\mathcal{H}_{\max }^{2}=\frac{1}{2|\vec{u}|_{0}^{2}\left|\zeta_{0}\right|^{2} \sin (\vartheta)}
$$

As can be seen in Fig. 6(a), this corresponds to a local maximum. At $\tau^{2}=$ $|\vec{u}|_{0}^{2}\left|\zeta_{0}\right|^{2} \cos (\vartheta), \mathcal{H}=0$; at even smaller proper times $\mathcal{H}^{2}<0$, which means that a local observer will have the impression that the Universe has entered an anti-de Sitter-like phase. Since we are in holomorphic gravity, there is no need to change the form of the line element (vielbein) Ansatz (79). The minimal expansion rate squared $\mathcal{H}_{\text {min }}^{2}$ is reached when $\tau=0$,

$$
\mathcal{H}_{\min }^{2}=-\frac{\cos (\vartheta)}{|\vec{u}|_{0}^{2}\left|\zeta_{0}\right|^{2}}
$$

which is singular only when $\vec{u}_{0}=0$, or equivalently when $u_{0}=1$, corresponding to a set of initial conditions of measure zero. Equations (122-124) nicely illustrate how one can relate the coordinate energy $E$ in (107) to the physical initial conditions of a point particle (observer), thus giving the physical meaning to the question: how large the holomorphic gravity corrections to general relativity actually are.

It is interesting to note that when $\cos (\vartheta)<0$, the expansion rate (124) becomes a global maximum, away from each $\mathcal{H}$ decreases monotonously in both directions, as can be seen in Fig. 6(b). This case represents a more conventional bounce Universe, and it is realized when the initial 3-force dominates over the initial 3-velocity, $G_{N}\left\|\vec{f}\left(\tau_{0}\right)\right\|>\left\|\vec{u}\left(\tau_{0}\right)\right\|$. 
Fig. 6 The physical Hubble parameter (122) in the curvature dominated epoch as a function of the proper time for different values of $\vartheta$ remains finite at all times. An infinite value of the physical Hubble parameter is attained when $\Im\left[\vec{u}_{0}\right]=0$, which represents a set of initial conditions of measure zero compared to the set of all initial conditions. (a) The physical Hubble parameter with initial conditions $\cos (\vartheta)<0$. (b) The physical Hubble parameter with initial conditions $\cos (\vartheta)>0$

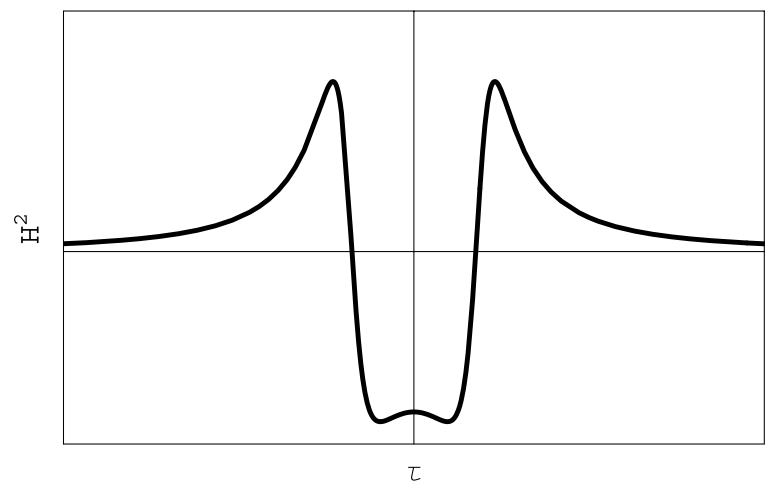

(a)

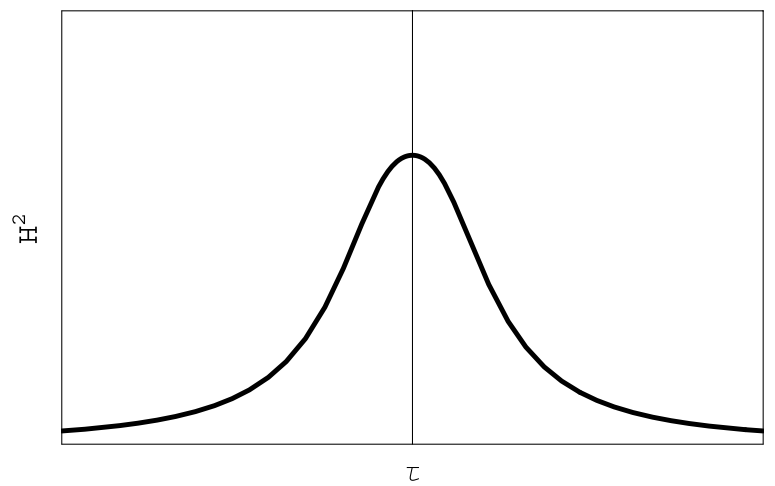

(b)

\subsubsection{Radiation Era}

Let us now consider radiation era $(\epsilon=2)$, in which case (117) integrates to,

$$
a \sqrt{a^{2}+U}-U \ln \left(a+\sqrt{a^{2}+U}\right)=\frac{\tau}{\zeta_{0}} \quad \text { (radiationera) }
$$

This transcendental equation cannot be solved for $a=a(\tau)$ in terms of elementary functions and thus it is hard to analyze in complete generality. A rather conclusive analysis can be, nevertheless, performed by integrating (115) numerically, which shows that, when $U$ and $\zeta_{0}$ are chosen real, the Hubble expansion rate $H^{2}=1 /\left(2 z^{0}\right)^{2}=1 /\left(2 \zeta_{0} a^{2}\right)^{2}$ becomes singular at $\tau=0$, as can be clearly seen in Fig. 7(a). When $U=\left(u^{0}\right)_{0}^{2}-1=\|\vec{u}\|_{0}^{2}$ is complex however, then-as can be seen in Fig. 7(b) - both $\mathfrak{R}\left[H^{2}\right]$ and $\Im\left[H^{2}\right]$ are in general nonzero, implying that the physical Hubble parameter remains finite at all times. We conclude that, just like in the case of curvature domination, there is no curvature singularity except for a set of initial conditions of measure zero $\left(\Im\left[u_{0}^{0}\right]=0\right)$, when compared to the set of all initial conditions ( $\Im\left[u_{0}^{0}\right]$ arbitrary). The Big Bang singularity is hence resolved also in the radiation era. We think that an analogous conclusion holds for more general expanding space-times. Even though the physics of radiation era is quite different from that 
Fig. 7 The physical Hubble parameter in radiation era $(\epsilon=2)$ as a function of proper time $\tau$ for different initial conditions. (a) The physical Hubble parameter for observers with real initial condition $U$ defined in (115) becomes singular at some time $\tau$, which corresponds to the Big Bang singularity. (b) When $U$ in (115) is complex, the physical Hubble parameter remains finite at all times, thus resolving the Big Bang singularity. The Hubble parameter is in general nonsymmetric under time reversal. Only when $U$ is purely imaginary, the physical Hubble parameter is symmetric under time reversal

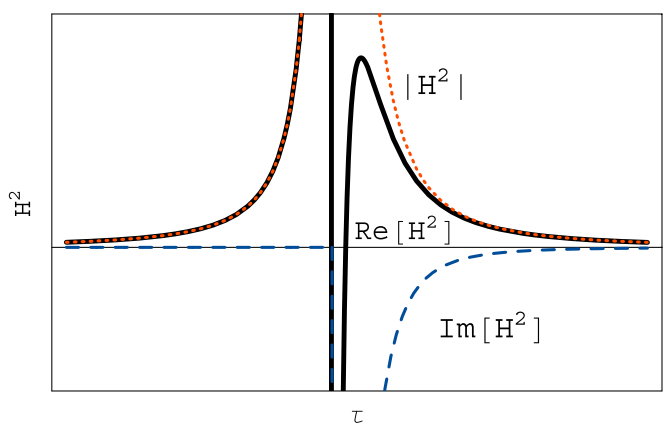

(a)

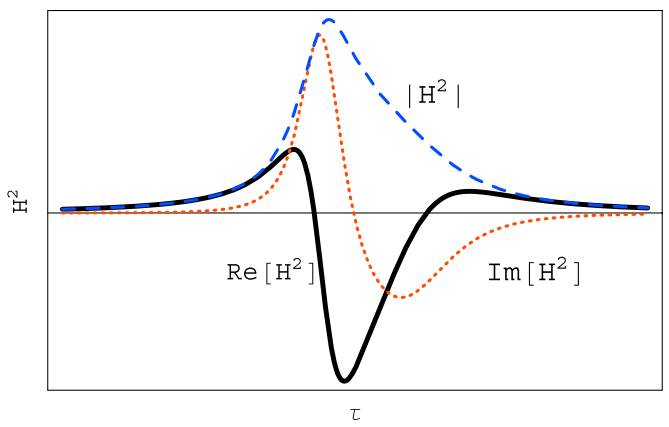

(b)

of Schwarzschild black holes, the corresponding geodesic equations (50) and (115) — based on which we performed the analyses of singularities - are of an identical form, and thus the conclusions of the analyses are quite similar.

\section{Conclusions and Discussion}

In this paper we propose a novel complex theory of gravity-holomorphic gravityand discussed various aspects of the theory: its weak field limit, Schwarzschild-like black holes, light deflection, gravitational waves and cosmology. Quite generically, holomorphic gravity predicts the general relativistic result plus an unobservably small correction. Exceptions are strong fields (close to the black whole and cosmological singularities), where large deviations from general relativity occur, and which act in favor of holomorphic gravity. For example, as opposed to general relativity, where singularities are generic, in holomorphic gravity they occur, but only for observers whose initial conditions correspond to a set of measure zero with respect to all possible initial conditions. This implies that a typical observer in holomorphic gravity will not experience any singularity, in the sense that its geodesic can be extended ad infinitum. It is reasonable to expect that quantization of space-time and momentum-energy will lead to further smearing of these point-like singularities.

Our original motivation for studying holomorphic gravity was in the fact that it is a simpler sister of Hermitian gravity [1], the latter being theoretically better moti- 
vated since it obeys Born's reciprocity symmetry. In Ref. [1] we found that Hermitian gravity resolves the cosmological Big Bang singularity in a similar fashion as holomorphic gravity. However, due to the complexity of Hermitian gravity, we were unable to construct a Hermitian black hole metric, and thus could not say anything definite about the strong field limit of Hermitian gravity near the black hole curvature singularity.

Albeit it is a geometric theory, holomorphic gravity is defined on an eight dimensional (phase-)space, and thus very different from general relativity. For this reason, we shall now discuss in some detail the physical interpretation of the additional four coordinates, which we refer to as the four momentum-energy $p^{\mu}$ of observer's frame, $y^{\mu}=\left(G_{N} / c^{3}\right) p^{\mu}$. Furthermore, we shall discuss in what sense, if at all, holomorphic gravity reduces to general relativity. In Einstein's theory the momentum-energy coordinates are tangent vectors on the geodesics of the manifold, $p_{G R}^{\mu}=m d u^{\mu} / d \tau$, where $\tau$ is an affine parameter (time) along the geodesic. This means that we can simultaneously specify the position and momentum of a particle. However, a well known fact is that in quantum mechanics the Heisenberg uncertainty principle forbids such a simultaneous measurement.

Mainly in order to address that difficulty within the context of gravity, in Ref. [1] we proposed a new theory-Hermitian gravity. Our hope was that Hermitian gravity would be a better quantum theory of gravity than Einstein's theory. Namely, Hermitian gravity incorporates two important ingredients: curved space and reciprocity symmetry, which is the symmetry obeyed by the commutation relations of quantum mechanics. This is achieved through the usage of Hermitian spaces, which contain four complex dimensions: four real dimensions correspond to the space-time coordinates and four imaginary dimensions to the momentum-energy coordinates. The hermitian structure of the coordinates on the Hermitian manifold and its relation to the Born's reciprocity symmetry (and to the commutation relations), justifies that interpretation.

Here we introduce holomorphic gravity, which is in many ways simpler then Hermitian gravity, but the classical theory has, mathematically speaking, a similar structure. The momentum-energy coordinates specify a frame in space-time-momentumenergy, which is described by a complex manifold. A test particle's kinematic is sensitive not only to its space-time coordinates, but also to its momentum-energy coordinates. Typical momentum-energy coordinates are so small that their effects are negligible, i.e. the change in momentum-energy will have only a tiny effect on the trajectory. To illustrate this, let us consider the line element of holomorphic gravity on flat space (20), and rewrite it as:

$$
\left(\frac{d s}{d \tau}\right)^{2}=-\left(c \frac{d t}{d \tau}\right)^{2}+\left(\frac{d \vec{x}}{d \tau}\right)^{2}+\frac{G_{N}^{2}}{c^{6}}\left[\left(\frac{d E}{c d \tau}\right)^{2}-\left(\frac{d \vec{p}}{d \tau}\right)^{2}\right]
$$

where $\tau$ is an affine time parameter along the geodesic. In the low energy limit, in which the four-force $f^{\mu}=d p^{\mu} / d \tau$ is small, this relation reduces to the on-shell relation of general relativity

$$
m^{2} c^{2} \approx-\left(m c \frac{d t}{d \tau}\right)^{2}+\left(m \frac{d \vec{x}}{d \tau}\right)^{2} \equiv-E_{G R}^{2}+\vec{p}_{G R}^{2}
$$


where we chose $d s / d \tau=c$. Hence we conclude, that the on-shell relation of general relativity is the low energy limit of holomorphic and Hermitian gravity theories. Thus in gravity theories on complex spaces it may be possible to represent the quantum mechanical off-shell paths of virtual particles by the geodesics on complex manifolds. In order to avoid possible misinterpretation, it is important to realize that, the momentum-energy coordinates on complex manifolds have, in general, nothing to do with the four-momenta of general relativity defined by the tangent vectors on geodesics. Nevertheless, we feel that the name is justified, given that in the low energy regime, in which the momentum-energy coordinates and the corresponding four-force are both small, the two notions of momentum-energy coincide.

Finally, a couple of remarks on the symmetries of holomorphic gravity are in order. The space-time-momentum-energy coordinates specify a frame. The frames of holomorphic gravity are related by a coordinate transformation belonging to the $S O(1,3 ; \mathbb{C})$ group. This group leaves the space-time-momentum-energy line element invariant, and is thus the symmetry of the theory. When the momentum-energy coordinates are small, the $S O(1,3 ; \mathbb{C})$ reduces to the Lorentz group $S O(1,3 ; \mathbb{R})$, which of course leaves the space-time element invariant. In its local disguise $\operatorname{SO}(1,3 ; \mathbb{C})$ generalises the diffeomorphism invariance of general relativity.

Acknowledgements The authors acknowledge financial support by FOM grant 07PR2522 and by Utrecht University.

Open Access This article is distributed under the terms of the Creative Commons Attribution Noncommercial License which permits any noncommercial use, distribution, and reproduction in any medium, provided the original author(s) and source are credited.

\section{References}

1. Mantz, C.L.M., Prokopec, T.: Hermitian gravity and cosmology. arXiv:0804.0213v1 [gr-qc]

2. Hawking, S.W., Ellis, G.F.R.: The Large Scale Structure of Space-Time. Cambridge University Press, Cambridge (1973)

3. Wald, R.M.: General Relativity. Chicago Univ. Pr., Chicago (1984) 491p

4. Einstein, A.: A generalization of the relativistic theory of gravitation. Ann. Math. 46, 578 (1945)

5. Einstein, A., Strauss, E.G.: A generalization of the relativistic theory of gravitation. 2. Ann. Math. 47, 731 (1946)

6. Moffat, J.W.: Generalized Riemann spaces. Math. Proc. Camb. Philos. Soc. 52, 623 (1956)

7. Moffat, J.W.: The foundations of a generalization of gravitation theory. Math. Proc. Camb. Philos. Soc. 53, 473 (1957)

8. Moffat, J.W.: The static spherically symmetric solutions in a unified field theory. Math. Proc. Camb. Philos. Soc. 53, 489 (1957)

9. Kerr, R.P.: On spherically symmetric solutions in Moffat's unified field theory. Il Nuovo Cimento 8, 789 (1958)

10. Moffat, J.W.: Nonsymmetric gravitational theory. Phys. Lett. B 355, 447 (1995). arXiv:gr-qc/9411006

11. Moffat, J.W.: Noncommutative quantum gravity. Phys. Lett. B 491, 345 (2000). arXiv:hep-th/0007181

12. Chamseddine, A.H.: Hermitian geometry and complex space-time. Commun. Math. Phys. 264, 291 (2006). arXiv:hep-th/0503048

13. Chamseddine, A.H.: Gravity in complex Hermitian space-time. arXiv:hep-th/0610099

14. Born, M.: A Suggestion For unifying quantum theory and relativity. R. Soc. Lond. Proc. Ser. A 165 (1938)

15. Born, M.: Reciprocity theory of elementary particles. Rev. Mod. Phys. 21, 463-473 (1949) 
16. Moffat, J.W.: Quantum gravity momentum representation and maximum invariant energy. arXiv:gr-qc/0401117

17. Low, S.G.: Reciprocal relativity of noninertial frames: quantum mechanics. J. Phys. A, Math. Theor. 40, 3999-4016 (2007). arXiv:math-ph/0606015

18. Hils, D., Hall, J.L.: Improved Kennedy-Thorndike experiment to test special relativity. Phys. Rev. Lett. 64, 1697 (1990)

19. Muller, H.: Testing Lorentz invariance by use of vacuum and matter filled cavity resonators. Phys. Rev. D 71, 045004 (2005). arXiv:hep-ph/0412385

20. Cane, F., et al.: Bound on Lorentz- and CPT-Violating Boost Effects for the Neutron. Phys. Rev. Lett. 93, 230801 (2004). arXiv:physics/0309070

21. Mantz, C.L.M.: Holomorphic gravity. Utrecht University Master's Thesis (2007). http://www1.phys. uu.nl/wwwitf/Teaching/2007/Mantz.pdf

22. Carroll, S.M.: Spacetime and Geometry: An Introduction to General Relativity. Addison-Wesley, San Francisco (2004) 513p

23. Mann, R.B., Moffat, J.W.: Linear approximation of a new theory of gravity. J. Phys. A 14, 2367 (1981)

24. Mann, R.B., Moffat, J.W.: Ghost properties of generalized theories of gravitation. Phys. Rev. D 26, 1858 (1982)

25. Kunstatter, G., Malzan, J., Moffat, J.W.: Geometrical interpretation of a generalized theory of gravitation. J. Math. Phys. 24, 886 (1983)

26. Hess, P.O., Greiner, W.: Pseudo-complex General Relativity. Int. J. Mod. Phys. E 18, 51 (2009). arXiv:0812.1738 [gr-qc]

27. Ratra, B., Peebles, P.J.E.: Cosmological consequences of a rolling homogeneous scalar field. Phys. Rev. D 37, 3406 (1988)

28. Joyce, M., Prokopec, T.: Turning around the sphaleron bound: electroweak baryogenesis in an alternative post-inflationary cosmology. Phys. Rev. D 57, 6022 (1998). arXiv:hep-ph/9709320 